\title{
Robust and Passive Constrained Fuzzy Control for Discrete Fuzzy Systems with Multiplicative Noises and Interval Time Delay
}

\author{
Wen-Jer Chang, ${ }^{1}$ Cheung-Chieh Ku, ${ }^{1}$ and Zong-Guo Fu ${ }^{2}$ \\ ${ }^{1}$ Department of Marine Engineering, National Taiwan Ocean University, Keelung 202, Taiwan \\ ${ }^{2}$ School of Naval Architecture and Marine Engineering, Zhejiang Ocean University, Zhoushan, Zhejiang 316022, China \\ Correspondence should be addressed to Wen-Jer Chang; wjchang@mail.ntou.edu.tw
}

Received 18 August 2013; Accepted 1 November 2013

Academic Editor: Baoyong Zhang

Copyright (C) 2013 Wen-Jer Chang et al. This is an open access article distributed under the Creative Commons Attribution License, which permits unrestricted use, distribution, and reproduction in any medium, provided the original work is properly cited.

\begin{abstract}
The passive fuzzy control for discrete-time uncertain Takagi-Sugeno (T-S) fuzzy models with multiplicative noises and time delay is investigated subject to robust asymptotical stability. Applying Jensen's inequality and free-weighting matrix technique, less conservative sufficient conditions are derived via choosing Lyapunov function to analyze and synthesize the robust asymptotical stability and passivity of closed-loop system. The derived conditions are not strictly linear matrix inequality (LMI) problems, thus the cone complementarity technique is employed to propose a suboptimal technique to solve the proposed nonstrictly LMI problems. An algorithm is developed in this paper to design the fuzzy controller which can be accomplished by state-feedback scheme or output-feedback scheme. Finally, numerical examples are provided to demonstrate the feasibility and applicability of the proposed fuzzy controller design technique.
\end{abstract}

\section{Introduction}

The delay-dependent stability analysis and synthesis of T-S fuzzy model with time delay has been extensively discussed in [1-4]. The time delay is an inherent and unavoidable effect on many practical dynamic nonlinear systems. In literature, the stability conditions of T-S fuzzy model with time delay were derived in terms of LMI problem [5] that can be solved by convex optimization technique. Furthermore, many relaxed techniques have been proposed to extend the maximum allowable delay range. For example, the piecewise Lyapunov function technique, fuzzy Lyapunov function approach, and free-weighting matrix approach have been used in [6-10] to reduce the conservatism of stability and stabilization problems for T-S fuzzy model with time delay. Although the conservatism of concerned problems can be decreased by such approaches, too many variables are needed to be found for satisfying their stability conditions. It is known that the complications of control synthesis and computational demands are increasing when the number of free variables is increased. Hence, the less conservative stability criteria with few free variables for dealing with T-S fuzzy model with time delay are worth to be discussed and investigated.

As well known, the performance requirement is the most important issue in the stability analysis and synthesis of control systems. For attenuating the effect of the external disturbance on systems, many efforts [11-17] proposed useful techniques such that the attenuation performance of system can be achieved. From [17], it can be found that the dissipativity and its particular case of passivity can be defined as $H_{\infty}$ performance constraint, positive real performance constraint, strictly input passive performance constraint, strictly output passive performance constraint, and strictly vary passive performance constraint by setting different power supply function [18]. Based on the power supply function, the passivity theory proposes a general and elastic tool for dealing with the effect of disturbance on the systems. On the other hand, uncertainty is often an existing phenomenon which is caused by modeling errors and internal perturbations. Generally, the parameter uncertainties of system are considered as norm-bounded time-varying function $[1,2,12]$. Considering 
external disturbances and uncertainties, the robust stability and passivity become important performances of control systems.

Recently, the stochastic systems have received much attention based on the stochastic modeling approach [19]. Therefore, many efforts have been devoted to expand the stability criteria [20,21] from deterministic systems to stochastic ones. Applying the fuzzy modeling approach, the nonlinear stochastic systems can be approximated by blending linear stochastic subsystems with corresponding membership functions. In literature [22-27], the nonlinear stochastic systems were represented by T-S fuzzy models in which the consequent part is structured by Itô stochastic differential equations. Since the consequent part of stochastic fuzzy mode belongs to linear stochastic systems, the Itô formula can also be employed to analyze the stability of stochastic T-S fuzzy systems. And then, the parallel distributed compensation (PDC) technique [17] was employed to design the fuzzy controller such that stability of nonlinear stochastic system is achieved. In case of continuous-time T-S fuzzy model, the delay-dependent stability and stabilization problems were studied in [23], robust fuzzy controller problems were discussed in [24-26], and robust fuzzy filtering design problem was addressed in [27, 28]. However, only few efforts [29-32] have been proposed for solving the stability and stabilization problems of discrete-time stochastic nonlinear systems.

From the above motivations, the fuzzy controller design of discrete uncertain T-S fuzzy model with multiplicative noise and time delay is investigated in this paper subject to passivity and robust asymptotical stability. The time delay effect is concerned as an interval time-varying delay [9] in this paper. Based on the discrete type Jensen inequality [33] and free-weighting matrix technique, the less conservative sufficient conditions are derived via Lyapunov function to achieve the robust asymptotical stability. In addition, the passivity theory is applied to discuss the external disturbance effect on the system. While deriving the conditions, none of the model transportation is used to avoid the potential conservatism of stability criteria in time delay systems. Since the proposed sufficient conditions belong to nonstrictly LMI problems, an algorithm based on cone complementarity technique [34] is developed in this paper. With the proposed algorithm, the feasible solutions of the conditions and allowable maximum upper bound of interval time-varying delay can be found by LMI technique. The main contributions of this paper can be summarized as follows. (1) Achieving passivity performance constraint, a robust fuzzy controller is developed in this paper for discrete uncertain T-S fuzzy model with multiplicative noise and time delay. (2) Comparing previous researches, the proposed fuzzy control method provides less conservatism because it can find bigger allowable maximum upper bound of time delay and its less desired unknown variables reduce the mathematical complexity. At last, two numerical examples are employed to demonstrate the effectiveness and application of the proposed design method.

Notation. The following notations are applied throughout this paper. The $\operatorname{tr}(\mathbf{A})$ denotes the trace of matrix A. The I is identity matrix with appropriate dimension. The $\operatorname{diag}\{\cdots\}$ means block-diagonal matrix. The ${ }^{*}$ denotes the transposed elements of matrices for symmetric position. The $E\{Q(\cdot)\}$ denotes the expected value of function $Q(\cdot)$. Moreover, let $\left(\Omega, \mathscr{F},\left\{\mathscr{F}_{t}\right\}_{t \geq 0}, \mathscr{P}\right)$ be a complete probability space with filtration $\left\{\mathscr{F}_{t}\right\}_{t \geq 0}$ satisfying the usual conditions (i.e., the filtration contains all $\mathscr{P}$-null sets and is right continuous).

\section{System Description and Problem Statement}

Applying the fuzzy modeling approach, the nonlinear stochastic systems can be represented by the T-S fuzzy model with multiplicative noise. Hence, the uncertain T-S fuzzy model with interval time-varying delay and multiplicative noise can be structured as follows:

$$
\begin{gathered}
x(k+1)=\sum_{i=1}^{r} h_{i}(\theta(k))\left\{f_{i}(k)+\bar{f}_{i}(k) \beta(k)\right\}, \\
z(k)=\sum_{i=1}^{r} h_{i}(\theta(k))\left\{\mathbf{C}_{1 i} x(k)+\mathbf{C}_{d i} x(k-\tau(k))+\mathbf{D}_{i} v(k)\right\}, \\
i=1,2, \ldots, r,
\end{gathered}
$$

where

$$
\begin{aligned}
f_{i}(k)= & \left(\mathbf{A}_{i}+\Delta \mathbf{A}_{i}\right) x(k)+\left(\mathbf{A}_{d i}+\Delta \mathbf{A}_{d i}\right) x(k-\tau(k)) \\
& +\left(\mathbf{B}_{i}+\Delta \mathbf{B}_{i}\right) u(k)+\left(\mathbf{E}_{i}+\Delta \mathbf{E}_{i}\right) v(k) \\
\bar{f}_{i}(k)= & \left(\overline{\mathbf{A}}_{i}+\bar{\Delta} \overline{\mathbf{A}}_{i}\right) x(k)+\left(\overline{\mathbf{A}}_{d i}+\bar{\Delta} \overline{\mathbf{A}}_{d i}\right) x(k-\tau(k)) \\
& +\left(\overline{\mathbf{B}}_{i}+\bar{\Delta} \overline{\mathbf{B}}_{i}\right) u(k)+\left(\overline{\mathbf{E}}_{i}+\bar{\Delta} \overline{\mathbf{E}}_{i}\right) v(k)
\end{aligned}
$$

Besides, $\sum_{i=1}^{r} h_{i}(\theta(k))=1, h_{i}(\theta(k)) \geq 0$ is the grade of membership function, $\theta(k)$ is the set of premise variables, $r$ is the number of fuzzy rules, and $\mathbf{A}_{i}, \mathbf{A}_{d i}, \mathbf{B}_{i}, \mathbf{E}_{i}, \overline{\mathbf{A}}_{i}, \overline{\mathbf{A}}_{d i}, \overline{\mathbf{B}}_{i}, \overline{\mathbf{E}}_{i}$, $\mathbf{C}_{1 i}, \mathbf{C}_{2 i}, \mathbf{C}_{d i}$ and $\mathbf{D}_{i}$ are known constant matrices with appropriate dimensions. $x(k) \in \mathfrak{R}^{n_{x}}, x(k-\tau(k)) \in$ $\mathfrak{R}^{n_{x}}, u(k) \in \mathfrak{R}^{n_{u}}, z(k) \in \mathfrak{R}^{n_{z}}, y(k) \in \mathfrak{R}^{n_{z}}, v(k) \in \mathfrak{R}^{n_{z}}$, and $\varphi(k)$ are state vector, state delay vector, controller input vector, controlled output vector, measurable output vector, external disturbance input vector, and initial condition, respectively. In addition, the $\beta(k)$ denotes standard scalar discrete Wiener process (Brownian motion) [19] on $(\Omega, \mathscr{F}, \mathscr{P})$ with $E\{\beta(k)\}=0$ and $E\left\{\beta^{2}(k)\right\}=1$. The time-varying delay $\tau(k)$ is a positive integer and satisfies $\tau_{\min } \leq \tau(k) \leq \tau_{\max }$. Here, $\tau_{\min }$ and $\tau_{\max }$ are known lower and upper bounds of delay, 
respectively. Moreover, $\Delta \mathbf{A}_{i}, \Delta \mathbf{A}_{d i}, \Delta \mathbf{B}_{i}, \Delta \mathbf{E}_{i}, \bar{\Delta} \overline{\mathbf{A}}_{i}, \bar{\Delta} \overline{\mathbf{A}}_{d i}$, $\bar{\Delta} \overline{\mathbf{B}}_{i}$, and $\bar{\Delta} \overline{\mathbf{E}}_{i}$ are defined as follows:

$$
\begin{aligned}
& {\left[\frac{\Delta \mathbf{A}_{i}}{\Delta} \overline{\mathbf{A}}_{i} \frac{\Delta \mathbf{A}_{d i}}{\Delta \overline{\mathbf{A}}_{d i}} \frac{\Delta \mathbf{B}_{i}}{\Delta} \overline{\mathbf{B}}_{i} \frac{\Delta \mathbf{E}_{i}}{\Delta} \overline{\mathbf{E}}_{i}\right]} \\
& =\left[\begin{array}{cc}
\mathbf{H}_{i} & 0 \\
0 & \overline{\mathbf{H}}_{i}
\end{array}\right]\left[\begin{array}{cc}
\Delta(k) & 0 \\
0 & \bar{\Delta}(k)
\end{array}\right]\left[\begin{array}{llll}
\mathbf{R}_{i} & \mathbf{R}_{d i} & \mathbf{R}_{B i} & \mathbf{R}_{E i} \\
\overline{\mathbf{R}}_{i} & \overline{\mathbf{R}}_{d i} & \overline{\mathbf{R}}_{B i} & \overline{\mathbf{R}}_{E i}
\end{array}\right],
\end{aligned}
$$

where $\mathbf{H}_{i}, \overline{\mathbf{H}}_{i}, \mathbf{R}_{i}, \mathbf{R}_{d i}, \mathbf{R}_{B i}, \mathbf{R}_{E i}, \overline{\mathbf{R}}_{i}, \overline{\mathbf{R}}_{d i}, \overline{\mathbf{R}}_{B i}$, and $\overline{\mathbf{R}}_{E i}$ are known constant matrices and $\Delta(k)$ and $\bar{\Delta}(k)$ are unknown time-varying function with $\Delta(k) \Delta(k) \leq \mathbf{I}$ and $\bar{\Delta}(k) \bar{\Delta}(k) \leq \mathbf{I}$. In the following statements, the $h_{i}(\theta(k)), \Delta(k)$, and $\bar{\Delta}(k)$ are denoted as $\widehat{h}_{i}, \Delta$, and $\bar{\Delta}$, respectively, for simplifying the context of this paper.

With state-feedback control scheme, the PDC-based fuzzy controller can be designed as follows:

$$
u(k)=\sum_{i=1}^{r} \widehat{h}_{i}\left(\mathbf{F}_{i} x(k)\right) .
$$

Substituting (4) into (1a), the closed-loop uncertain T-S fuzzy model with interval time-varying delay and multiplicative noise can be inferred as follows:

$$
\begin{array}{r}
x(k+1) \\
=\sum_{i=1, j=1}^{r} \widehat{h}_{i} \widehat{h}_{j}\left\{\left(\mathbf{f}_{i j}+\mathbf{H}_{i} \Delta \mathbf{f}_{R i j}+\left(\overline{\mathbf{f}}_{i j}+\overline{\mathbf{H}}_{i} \bar{\Delta} \overline{\mathbf{f}}_{R i j}\right) \beta(k)\right) \xi(k)\right\} \\
=\sum_{i=1}^{r} \sum_{j=1}^{r} \widehat{h}_{i} \widehat{h}_{j}\left\{\frac { 1 } { 2 } \left(\mathbf{f}_{i j}+\mathbf{H}_{i} \Delta \mathbf{f}_{R i j}+\mathbf{f}_{j i}+\mathbf{H}_{j} \Delta \mathbf{f}_{R j i}\right.\right. \\
\left.\left.+\left(\overline{\mathbf{f}}_{i j}+\overline{\mathbf{H}}_{i} \bar{\Delta} \overline{\mathbf{f}}_{R i j}+\overline{\mathbf{f}}_{j i}+\overline{\mathbf{H}}_{j} \bar{\Delta} \overline{\mathbf{f}}_{R j i}\right) \beta(k)\right) \xi(k)\right\},
\end{array}
$$

where

$$
\begin{aligned}
& \xi(k)
\end{aligned}
$$

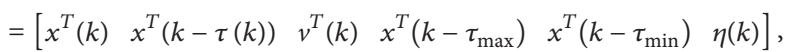

$$
\begin{aligned}
& \mathbf{f}_{i j}=\left[\begin{array}{llll}
\mathbf{G}_{i j} & \mathbf{A}_{d i} & \mathbf{E}_{i} & 0_{1 \times 3}
\end{array}\right], \\
& \overline{\mathbf{f}}_{i j}=\left[\begin{array}{llll}
\overline{\mathbf{G}}_{i j} & \overline{\mathbf{A}}_{d i} & \overline{\mathbf{E}}_{i} & 0_{1 \times 3}
\end{array}\right], \\
& \mathbf{f}_{R i j}=\left[\begin{array}{llll}
\mathbf{R}_{i j} & \mathbf{R}_{d i} & \mathbf{R}_{E i} & 0_{1 \times 3}
\end{array}\right], \\
& \overline{\mathbf{f}}_{R i j}=\left[\begin{array}{llll}
\overline{\mathbf{R}}_{i j} & \overline{\mathbf{R}}_{d i} & \overline{\mathbf{R}}_{E i} & 0_{1 \times 3}
\end{array}\right], \\
& \mathbf{G}_{i j}=\mathbf{A}_{i}+\mathbf{B}_{i} \mathbf{F}_{j}, \quad \overline{\mathbf{G}}_{i j}=\overline{\mathbf{A}}_{i}+\overline{\mathbf{B}}_{i} \mathbf{F}_{j}, \\
& \mathbf{R}_{i j}=\mathbf{R}_{i}+\mathbf{R}_{B i} \mathbf{F}_{j}, \quad \overline{\mathbf{R}}_{i j}=\overline{\mathbf{R}}_{i}+\overline{\mathbf{R}}_{B i} \mathbf{F}_{j}, \\
& \eta(k)=x(k)-x(k-\tau(k)) \text {. }
\end{aligned}
$$

For deriving the stability criteria of this paper, the following definitions and lemmas are necessary to be introduced. Based on the energy concept, the passivity theory provides a useful tool to discuss the effect of external disturbance for achieving attenuation performance. Here, the passivity property is introduced in the following definition.

Definition 1 (see [18]). If there exist constant matrices $\mathbf{S}_{1}$, $\mathbf{S}_{2}$, and $\mathbf{S}_{3}$ for satisfying the following inequality, then the closed-loop system (5) is called passive with the disturbance $v(k)$ and controlled output $z(k)$ for all terminal time $k_{q}>0$ :

$$
\begin{aligned}
& E\left\{2 \sum_{k=0}^{k_{q}} z^{T}(k) \mathbf{S}_{1} v(k)\right\} \\
& >E\left\{\sum_{k=0}^{k_{q}} z^{T}(k) \mathbf{S}_{2} z(k)+\sum_{k=0}^{k_{q}} v^{T}(k) \mathbf{S}_{3} v(k)\right\} .
\end{aligned}
$$

Via the well-known mathematical definition of power supply function [18], the passivity theory includes several performance constraints with setting matrices $\mathbf{S}_{1}, \mathbf{S}_{2}$, and $\mathbf{S}_{3}$. In this paper, the generalized power supply function (7) is proposed to be the constraint index. Besides, for illustrating the concerned stability concept clearly, the following definition is introduced.

Definition 2 (see [19]). For the closed-loop system (5) without external disturbance input, that is, $v(k)=0$, the solution with admissible uncertainties is robustly asymptotically stable in the mean square if $E\{x(k)\}$ and state correlation matrix $E\left\{x^{T}(k) x(k)\right\}$ converge to zero as $t \rightarrow \infty$.

For analyzing the uncertainties of systems, the following lemma is proposed to convert the uncertain matrices into deterministic matrices.

Lemma 3 (see [26]). Given real compatible dimension matrices $\mathbf{A}, \mathbf{H}$, and $\mathbf{R}$ for any matrix $\mathbf{X}>0, \varepsilon>0, \Delta$ with $\Delta^{T} \Delta \leq \mathbf{I}$, one can find the following results:

$$
\begin{aligned}
(\mathbf{A}+\mathbf{H} & \Delta \mathbf{R})^{T} \mathbf{X}(\mathbf{A}+\mathbf{H} \Delta \mathbf{R}) \\
& \leq \mathbf{A}^{T}\left(\mathbf{X}^{-1}-\varepsilon \mathbf{H} \mathbf{H}^{T}\right)^{-1} \mathbf{A}+\varepsilon^{-1} \mathbf{R}^{T} \mathbf{R},
\end{aligned}
$$

where $\mathbf{X}^{-1}-\varepsilon \mathbf{H H}^{T}>0$.

In this paper, the following discrete type Jensen inequality is employed to derive the less conservative sufficient conditions.

Lemma 4 (see [33]). For any compatible constant matrices $\mathbf{Q}=\mathbf{Q}^{T}>0$, scalars $\tau_{\min }>0$ and $\tau_{\max }>0$ satisfying $\tau_{\min }<$ $\tau_{\max }$ and vector function $\emptyset:\left[\tau_{\min }, \tau_{\min }+1, \ldots, \tau_{\max }\right] \rightarrow$ $\mathfrak{R}^{n_{x}}$ such that the following sums are well defined, it holds that

$$
\begin{gathered}
-\left(\tau_{\max }-\tau_{\min }+1\right) \sum_{k=\tau_{\min }}^{\tau_{\max }} \omega^{T}(k) \mathbf{Q} \omega(k) \\
\leq-\left(\sum_{k=\tau_{\min }}^{\tau_{\max }} \omega(k)\right)^{T} \mathbf{Q} \sum_{k=\tau_{\min }}^{\tau_{\max }} \omega(k) .
\end{gathered}
$$


From the above definitions and lemmas, the sufficient conditions are derived in the following section for guaranteeing the robust asymptotical stability and passivity of closedloop system (5).

\section{Stabilization Criteria and Robust Fuzzy Controller Design}

In this section, the stability criteria for closed-loop system (5) are derived with both of state-feedback control scheme and output-feedback control scheme. The sufficient conditions derived in this paper are nonstrictly LMI problems. In order to solve the proposed nonstrictly LMI problems, an algorithm is also developed in this section.

Theorem 5. Given performance parameters $\mathbf{S}_{1}, \mathbf{S}_{2} \geq 0$, and $\mathbf{S}_{3}$; positive scalars $\tau_{\min }>0$ and $\tau_{\max }>0$, if there exist positive definite matrices $\mathbf{P}_{1}>0, \mathbf{P}_{2}>0, \mathbf{P}_{3}>0, \mathbf{P}_{4}>$ 0 , and $\mathbf{P}_{5}>0$, any matrices $\mathbf{N}_{1}, \mathbf{N}_{2}$, and $\mathbf{N}_{3}$, scalars $\varepsilon_{i j}>$ 0 and $\bar{\varepsilon}_{i j}>0$, and state-feedback gains $\mathbf{F}_{i}$ for satisfying the following conditions, then the closed-loop system (5) is passive and robustly asymptotically stable in the mean square:

$$
\Phi<0 \quad \text { for } i, j \leq r,
$$

where

$$
\begin{gathered}
\boldsymbol{\Phi}=\left[\begin{array}{ccccc}
\boldsymbol{\Phi}_{11 i j} & \boldsymbol{\Phi}_{12} & \boldsymbol{\Phi}_{13} & \boldsymbol{\Phi}_{14} & \boldsymbol{\Phi}_{15} \\
* & \boldsymbol{\Phi}_{22} & 0 & 0 & 0 \\
* & * & \boldsymbol{\Phi}_{33} & 0 & 0 \\
* & * & * & \boldsymbol{\Phi}_{44} & 0 \\
* & * & * & * & \boldsymbol{\Phi}_{55}
\end{array}\right], \\
\boldsymbol{\Phi}_{11 i j}=\overline{\boldsymbol{\Phi}}+\frac{1}{2}\left(\mathbf{W}_{i j}+\mathbf{W}_{j i}\right), \\
\boldsymbol{\Phi}_{12}=\frac{1}{2}\left[\boldsymbol{\Phi}_{12 i j}\right. \\
\left.\boldsymbol{\Phi}_{12 j i}\right], \quad \boldsymbol{\Phi}_{13}=\sqrt{\tau_{\max }} \boldsymbol{\Phi}_{12}, \\
\boldsymbol{\Phi}_{14}=\frac{1}{2}\left[\boldsymbol{\Phi}_{14 i j} \boldsymbol{\Phi}_{14 j i}\right], \quad \boldsymbol{\Phi}_{15}=\sqrt{\tau_{\max }} \boldsymbol{\Phi}_{14}, \\
\boldsymbol{\Phi}_{22}=\operatorname{diag}\left\{\boldsymbol{\Phi}_{22 i j}, \boldsymbol{\Phi}_{22 j i}\right\}, \quad \boldsymbol{\Phi}_{33}=\operatorname{diag}\left\{\boldsymbol{\Phi}_{33 i j}, \boldsymbol{\Phi}_{33 j i}\right\}, \\
\boldsymbol{\Phi}_{44}=\operatorname{diag}\left\{\boldsymbol{\Phi}_{44 i j}, \boldsymbol{\Phi}_{44 j i}\right\}, \quad \boldsymbol{\Phi}_{55}=\operatorname{diag}\left\{\boldsymbol{\Phi}_{55 i j}, \boldsymbol{\Phi}_{55 j i}\right\},
\end{gathered}
$$

$\bar{\Phi}$

$$
=\left[\begin{array}{cccccc}
\overline{\mathbf{\Phi}}_{11} & \tau_{\max }^{-1} \mathbf{P}_{5}-\mathbf{N}_{1}+\mathbf{N}_{2}^{T} & 0 & 0 & 0 & -\mathbf{N}_{1}+\mathbf{N}_{3}^{T} \\
* & -\tau_{\max }^{-1} \mathbf{P}_{5}-\mathbf{P}_{4}-\mathbf{N}_{2}^{T}-\mathbf{N}_{2} & 0 & 0 & 0 & -\mathbf{N}_{2}-\mathbf{N}_{3}^{T} \\
* & * & 0 & 0 & 0 & 0 \\
* & * & * & -\mathbf{P}_{2} & 0 & 0 \\
* & * & * & * & -\mathbf{P}_{3} & 0 \\
* & * & * & * & * & -\mathbf{N}_{3}^{T}-\mathbf{N}_{3}
\end{array}\right],
$$

$$
=\left[\begin{array}{cccccc}
\mathbf{C}_{1 i}^{T} \mathbf{S}_{2} \mathbf{C}_{1 j} & \mathbf{C}_{1 i}^{T} \mathbf{S}_{2} \mathbf{C}_{d j} & \mathbf{C}_{1 i}^{T} \mathbf{S}_{2} \mathbf{D}_{j}-\mathbf{C}_{1 i}^{T} \mathbf{S}_{1} & 0 & 0 & 0 \\
* & \mathbf{C}_{d i}^{T} \mathbf{S}_{2} \mathbf{C}_{d j} & \mathbf{C}_{d i}^{T} \mathbf{S}_{2} \mathbf{D}_{j}-\mathbf{C}_{d i}^{T} \mathbf{S}_{1} & 0 & 0 & 0 \\
* & * & \mathbf{S}_{3}-\mathbf{D}_{i}^{T} \mathbf{S}_{1}-\mathbf{S}_{1}^{T} \mathbf{D}_{i}+\mathbf{D}_{i}^{T} \mathbf{S}_{2} \mathbf{D}_{j} & 0 & 0 & 0 \\
* & * & * & 0 & 0 & 0 \\
* & * & * & * & 0 & 0 \\
* & * & * & * & * & 0
\end{array}\right],
$$

$$
\begin{aligned}
& \overline{\mathbf{\Phi}}_{11}=\mathbf{P}_{2}+\mathbf{P}_{3}+\left(\tau_{\max }-\tau_{\min }+1\right) \mathbf{P}_{4}-\mathbf{P}_{1}-\tau_{\max }^{-1} \mathbf{P}_{5} \\
& +\mathbf{N}_{1}^{T}+\mathbf{N}_{1} \\
& \boldsymbol{\Phi}_{12 i j}=\left[\begin{array}{ll}
\mathbf{f}_{i j}^{T} & \mathbf{f}_{R i j}^{T}
\end{array}\right], \quad \boldsymbol{\Phi}_{14 i j}=\left[\begin{array}{ll}
\overline{\mathbf{f}}_{i j}^{T} & \overline{\mathbf{f}}_{R i j}^{T}
\end{array}\right], \\
& \boldsymbol{\Phi}_{22 i j}=\operatorname{diag}\left\{\varepsilon_{i j} \mathbf{H}_{i} \mathbf{H}_{i}^{T}-\mathbf{P}_{1}^{-1},-\varepsilon_{i j} \mathbf{I}\right\}, \\
& \boldsymbol{\Phi}_{33 i j}=\operatorname{diag}\left\{\varepsilon_{i j} \mathbf{H}_{i} \mathbf{H}_{i}^{T}-\mathbf{P}_{5}^{-1},-\varepsilon_{i j} \mathbf{I}\right\}, \\
& \boldsymbol{\Phi}_{44 i j}=\operatorname{diag}\left\{\bar{\varepsilon}_{i j} \overline{\mathbf{H}}_{i} \overline{\mathbf{H}}_{i}^{T}-\mathbf{P}_{1}^{-1},-\bar{\varepsilon}_{i j} \mathbf{I}\right\}, \\
& \boldsymbol{\Phi}_{55 i j}=\operatorname{diag}\left\{\bar{\varepsilon}_{i j} \overline{\mathbf{H}}_{i} \overline{\mathbf{H}}_{i}^{T}-\mathbf{P}_{5}^{-1},-\bar{\varepsilon}_{i j} \mathbf{I}\right\} \text {. }
\end{aligned}
$$

Proof. Choose the following Lyapunov function:

$$
V(x(k))=V_{1}(x(k))+V_{2}(x(k))+V_{3}(x(k))+V_{4}(x(k)) \text {, }
$$

where

$$
\begin{gathered}
V_{1}(x(k))=x^{T}(k) \mathbf{P}_{1} x(k) \\
V_{2}(x(k))=\sum_{\ell=k-\tau_{\max }}^{k-1} x^{T}(\ell) \mathbf{P}_{2} x(\ell)+\sum_{\ell=k-\tau_{\min }}^{k-1} x^{T}(\ell) \mathbf{P}_{3} x(\ell) \\
+\sum_{\ell=k-\tau(k)}^{k-1} x^{T}(\ell) \mathbf{P}_{4} x(\ell) \\
V_{3}(x(k))=\sum_{s=-\tau_{\max }+1} \sum_{\ell=k+s}^{k-1} x^{T}(\ell) \mathbf{P}_{4} x(\ell) \\
V_{4}(x(k))=\sum_{s=-\tau_{\max }+1}^{-1} \sum_{\ell=k+s}^{k-1} \Delta x^{T}(\ell) \mathbf{P}_{5} \Delta x(\ell)
\end{gathered}
$$

where $\Delta x(k)=x(k+1)-x(k)$. Firstly, calculating the difference of $V_{1}(x(k))$ in (14a) along the trajectory of closedloop system (5) and taking the mathematical expectation, the following inequality can be obtained with relations $\widehat{h}_{i} \widehat{h}_{j} \widehat{h}_{m} \widehat{h}_{n} \leq \widehat{h}_{i} \widehat{h}_{j}$ and $0 \leq \widehat{h}_{i} \leq 1$ :

$$
\begin{aligned}
& E\left\{\Delta V_{1}(x(k))\right\} \\
& \leq E\left\{\sum_{i=1}^{r} \sum_{j=1}^{r} \widehat{h}_{i} \widehat{h}_{j}\right. \\
& \quad \times\left\{\xi ^ { T } ( k ) \left(\left(\mathbf{f}+\mathbf{H} \widetilde{\Delta} \mathbf{f}_{R}\right)^{T} \widetilde{\mathbf{P}}_{1}\left(\mathbf{f}+\mathbf{H} \widetilde{\Delta} \mathbf{f}_{R}\right)\right.\right. \\
& +\left(\overline{\mathbf{f}}+\overline{\mathbf{H}} \widetilde{\bar{\Delta}} \overline{\mathbf{f}}_{R}\right)^{T} \times \widetilde{\mathbf{P}}_{1}\left(\overline{\mathbf{f}}+\overline{\mathbf{H}} \widetilde{\left.\left.\bar{\Delta} \mathbf{f}_{R}\right)\right)}\right. \\
& \left.\left.\times \xi(k)-x^{T}(k) \mathbf{P}_{1} x(k)\right\}\right\},
\end{aligned}
$$


where $\mathbf{f}=(1 / 2)\left[\begin{array}{ll}\mathbf{f}_{i j}^{T} & \mathbf{f}_{j i}^{T}\end{array}\right]^{T}, \mathbf{f}_{R}=(1 / 2)\left[\begin{array}{ll}\mathbf{f}_{R i j}^{T} & \mathbf{f}_{R j i}^{T}\end{array}\right]^{T}, \overline{\mathbf{f}}=$ $(1 / 2)\left[\begin{array}{ll}\overline{\mathbf{f}}_{i j}^{T} & \overline{\mathbf{f}}_{j i}^{T}\end{array}\right]^{T}, \overline{\mathbf{f}}_{R}=(1 / 2)\left[\begin{array}{ll}\overline{\mathbf{f}}_{R i j}^{T} & \overline{\mathbf{f}}_{R j i}^{T}\end{array}\right]^{T}, \mathbf{H}=\operatorname{diag}\left\{\mathbf{H}_{i}, \mathbf{H}_{j}\right\}$, $\overline{\mathbf{H}}=\operatorname{diag}\left\{\overline{\mathbf{H}}_{i}, \overline{\mathbf{H}}_{j}\right\}, \widetilde{\Delta}=\operatorname{diag}\{\Delta, \Delta\}, \widetilde{\bar{\Delta}}=\operatorname{diag}\{\bar{\Delta}, \bar{\Delta}\}$, and $\widetilde{\mathbf{P}}_{1}=$ $\operatorname{diag}\left\{\mathbf{P}_{1}, \mathbf{P}_{1}\right\}$. According to Lemma 3 , the following inequalities always hold:

$$
\begin{aligned}
(\mathbf{f}+ & \left.\mathbf{H} \widetilde{\Delta} \mathbf{f}_{R}\right)^{T} \widetilde{\mathbf{P}}_{1}\left(\mathbf{f}+\mathbf{H} \widetilde{\Delta} \mathbf{f}_{R}\right) \\
& \leq \mathbf{f}^{T}\left(\widetilde{\mathbf{P}}_{1}^{-1}-\widetilde{\varepsilon} \mathbf{H} \mathbf{H}\right)^{-1} \mathbf{f}+\widetilde{\mathcal{\varepsilon}}^{-1} \mathbf{f}_{R}^{T} \mathbf{f}_{R}, \\
(\overline{\mathbf{f}}+\overline{\mathbf{H}} & \left.\widetilde{\bar{\Delta}} \overline{\mathbf{f}}_{R}\right)^{T} \widetilde{\mathbf{P}}_{1}\left(\overline{\mathbf{f}}+\overline{\mathbf{H}} \widetilde{\bar{\Delta}} \overline{\mathbf{f}}_{R}\right) \\
& \leq \overline{\mathbf{f}}^{T}\left(\widetilde{\mathbf{P}}_{1}^{-1}-\widetilde{\bar{\varepsilon}} \overline{\mathbf{H}} \overline{\mathbf{H}}\right)^{-1} \overline{\mathbf{f}}+\widetilde{\bar{\varepsilon}}^{-1} \overline{\mathbf{f}}_{R}^{T} \overline{\mathbf{f}}_{R},
\end{aligned}
$$

where $\widetilde{\varepsilon}=\operatorname{diag}\left\{\varepsilon_{i j}, \varepsilon_{j i}\right\}$ and $\widetilde{\bar{\varepsilon}}=\operatorname{diag}\left\{\bar{\varepsilon}_{i j}, \bar{\varepsilon}_{j i}\right\}$. According to (16a) and (16b), one can obtain the following inequality from (15):

$$
\begin{aligned}
& E\left\{\Delta V_{1}(x(k))\right\} \\
& \leq E\left\{\sum_{i=1}^{r} \sum_{j=1}^{r} \widehat{h}_{i} \widehat{h}_{j}\right. \\
& \qquad\left\{\xi ^ { T } ( k ) \left(\mathbf{f}^{T}\left(\widetilde{\mathbf{P}}_{1}^{-1}-\widetilde{\varepsilon} \mathbf{H} \mathbf{H}\right)^{-1} \mathbf{f}\right.\right. \\
& +\widetilde{\varepsilon}^{-1} \mathbf{f}_{R}^{T} \mathbf{f}_{R}+\overline{\mathbf{f}}^{T}\left(\widetilde{\mathbf{P}}_{1}^{-1}-\widetilde{\bar{\varepsilon}} \overline{\mathbf{H}} \overline{\mathbf{H}}\right)^{-1} \overline{\mathbf{f}} \\
& \\
& \left.\left.\left.\quad+\widetilde{\bar{\varepsilon}}^{-1} \overline{\mathbf{f}}_{R}^{T} \overline{\mathbf{f}}_{R}\right) \xi(k)-x^{T}(k) \mathbf{P}_{1} x(k)\right\}\right\} .
\end{aligned}
$$

Furthermore, the difference of other Lyapunov functions, that is, $V_{2}(x(k)), V_{3}(x(k))$, and $V_{4}(x(k))$, can be obtained as follows:

$$
\begin{aligned}
& E\left\{\Delta V_{2}(x(k))\right\} \\
& \leq E\left\{x^{T}(k)\left(\mathbf{P}_{2}+\mathbf{P}_{3}+\mathbf{P}_{4}\right) x(k)\right. \\
& \quad-x^{T}\left(k-\tau_{\max }\right) \mathbf{P}_{2} x\left(k-\tau_{\max }\right)-x^{T}\left(k-\tau_{\min }\right) \\
& \quad \times \mathbf{P}_{3} x\left(k-\tau_{\min }\right)-x^{T}(k-\tau(k)) \mathbf{P}_{4} x(k-\tau(k)) \\
& \left.\quad+\sum_{s=k-\tau_{\max }+1}^{k-\tau_{\min }} x^{T}(s) \mathbf{P}_{4} x(s)\right\}
\end{aligned}
$$

$E\left\{\Delta V_{3}(x(k))\right\}$$$
=E\left\{\left(\tau_{\max }-\tau_{\min }\right) x^{T}(k) \mathbf{P}_{4} x(k)-\sum_{s=k-\tau_{\max }+1}^{k-\tau_{\min }} x^{T}(s) \mathbf{P}_{4} x(s)\right\},
$$

$$
\begin{aligned}
& E\left\{\Delta V_{4}(x(k))\right\} \\
& =E\left\{\tau_{\max }(x(k+1)-x(k))^{T} \mathbf{P}_{5}(x(k+1)-x(k))\right. \\
& \left.\quad-\sum_{s=-\tau_{\max }+1}^{-1} \Delta x^{T}(s) \mathbf{P}_{5} \Delta x(s)\right\} .
\end{aligned}
$$

Substituting (5) into the first term of the right-hand side of (20) and using the relation $h_{i} h_{j} h_{m} h_{n} \leq h_{i} h_{j}$, one has

$$
\begin{aligned}
& E\left\{\tau_{\max }(x(k+1)-x(k))^{T} \mathbf{P}_{5}(x(k+1)-x(k))\right\} \\
& \leq E\left\{\sum_{i=1}^{r} \sum_{j=1}^{r} \widehat{h}_{i} \widehat{h}_{j} \tau_{\max }\right. \\
& \quad \times\left\{\xi^{T}(k)\left(\left(\mathbf{f}-\mathbf{I}_{2}\right)+\mathbf{H} \widetilde{\Delta} \mathbf{f}_{R}\right)^{T} \widetilde{\mathbf{P}}_{5}\right. \\
& \times\left(\left(\mathbf{f}-\mathbf{I}_{2}\right)+\mathbf{H} \widetilde{\Delta} \mathbf{f}_{R}\right)+\left(\overline{\mathbf{f}}+\overline{\mathbf{H}} \widetilde{\bar{\Delta}} \overline{\mathbf{f}}_{R}\right)^{T} \\
& \\
& \left.\left.\left.\times \widetilde{\mathbf{P}}_{5}\left(\overline{\mathbf{f}}+\overline{\mathbf{H}} \widetilde{\bar{\Delta}} \overline{\mathbf{f}}_{R}\right)\right) \xi(k)\right\}\right\},
\end{aligned}
$$

where $\widetilde{\mathbf{P}}_{5}=\operatorname{diag}\left\{\mathbf{P}_{5}, \mathbf{P}_{5}\right\}, \mathbf{I}_{2}=\left[\begin{array}{l}\mathbf{I}_{1} \\ \mathbf{I}_{1}\end{array}\right]$, and $\mathbf{I}_{1}=\left[\begin{array}{llllll}\mathbf{I} & 0 & 0 & 0 & 0 & 0\end{array}\right]$. With the similar relations from (15) to (17), one can also obtain the following inequality:

$$
\begin{aligned}
E\left\{\tau_{\max }(x(k+1)-x(k))^{T} \mathbf{P}_{5}(x(k+1)-x(k))\right\} & \\
\leq E\left\{\sum_{i=1}^{r} \sum_{j=1}^{r} \widehat{h}_{i} \widehat{h}_{j} \tau_{\max }\right. & \\
& \times\left\{\xi^{T}(k)\right. \\
& \left(\left(\mathbf{f}-\mathbf{I}_{2}\right)^{T}\right. \\
& \times\left(\widetilde{\mathbf{P}}_{5}^{-1}-\widetilde{\mathcal{E}} \mathbf{H} \mathbf{H}\right)^{-1}\left(\mathbf{f}-\mathbf{I}_{2}\right)+\widetilde{\mathcal{\varepsilon}}^{-1} \mathbf{f}_{R}^{T} \mathbf{f}_{R} \\
& +\overline{\mathbf{f}}^{T}\left(\widetilde{\mathbf{P}}_{5}^{-1}-\widetilde{\bar{\varepsilon}} \overline{\mathbf{H}} \overline{\mathbf{H}}\right)^{-1} \overline{\mathbf{f}} \\
& \left.\left.\left.+\widetilde{\bar{\varepsilon}}^{-1} \overline{\mathbf{f}}_{R}^{T} \overline{\mathbf{f}}_{R}\right) \xi(k)\right\}\right\} .
\end{aligned}
$$

On the other hand, using Lemma 4, one has the following relation from the second term of the right-hand side of (20):

$$
\begin{aligned}
& -\sum_{s=-\tau_{\max }+1}^{-1} \Delta x^{T}(s) \mathbf{P}_{5} \Delta x(s) \\
& \quad \leq-\tau_{\max }^{-1}\left(\sum_{s=-\tau(k)+1}^{-1} \Delta x(s)\right)^{T} \mathbf{P}_{5}\left(\sum_{s=-\tau(k)+1}^{-1} \Delta x(s)\right) \\
& \quad=-\tau_{\max }^{-1}(x(k)-x(k-\tau(k)))^{T} \mathbf{P}_{5}(x(k)-x(k-\tau(k))) .
\end{aligned}
$$


Thus, the following inequality can be obtained due to (22) and (23):

$$
\begin{aligned}
& E\left\{\Delta V_{4}(x(k))\right\} \\
& \begin{array}{l}
\leq E\left\{\sum_{i=1}^{r} \sum_{j=1}^{r} \widehat{h}_{i} \widehat{h}_{j} \tau_{\max }\right. \\
\quad \times\left\{\xi ^ { T } ( k ) \left(\left(\mathbf{f}-\mathbf{I}_{2}\right)^{T}\left(\widetilde{\mathbf{P}}_{5}^{-1}-\widetilde{\varepsilon} \mathbf{H} \mathbf{H}\right)^{-1}\left(\mathbf{f}-\mathbf{I}_{2}\right)\right.\right. \\
+\widetilde{\varepsilon}^{-1} \mathbf{f}_{R}^{T} \mathbf{f}_{R}+\overline{\mathbf{f}}^{T}\left(\widetilde{\mathbf{P}}_{5}^{-1}-\widetilde{\bar{\varepsilon}} \overline{\mathbf{H}} \overline{\mathbf{H}}\right)^{-1} \overline{\mathbf{f}} \\
\left.\left.\left.\quad+\widetilde{\bar{\varepsilon}}^{-1} \overline{\mathbf{f}}_{R}^{T} \overline{\mathbf{f}}_{R}\right) \xi(k)\right\}\right\} \\
-\tau_{\max }^{-1}(x(k)-x(k-\tau(k)))^{T} \times \mathbf{P}_{5}(x(k)-x(k-\tau(k))) .
\end{array}
\end{aligned}
$$

For reducing the conservatism, the following equation is introduced with the concept of free-weighting matrices technique [10]. For any matrices with approximate dimensions, the following equation always holds:

$$
\begin{gathered}
2\left(x^{T}(k) \mathbf{N}_{1}+x^{T}(k-\tau(k)) \mathbf{N}_{2}+\eta^{T}(k) \mathbf{N}_{3}\right) \\
\times(x(k)-x(k-\tau(k))-\eta(k))=0 .
\end{gathered}
$$

From (17), (18), (19), (24), and (25), one has

$$
\begin{aligned}
& E\{\Delta V(x(k))\} \\
& =E\left\{\Delta V_{1}(x(k))+\Delta V_{2}(x(k))+\Delta V_{3}(x(k))+\Delta V_{4}(x(k))\right\} \\
& \leq E\left\{\sum_{i=1}^{r} \sum_{j=1}^{r} \widehat{h}_{i} \widehat{h}_{j}\left\{\xi^{T}(k) \Pi_{i j} \xi(k)\right\}\right\},
\end{aligned}
$$

where

$$
\begin{aligned}
\boldsymbol{\Pi}_{i j}=\overline{\boldsymbol{\Phi}}-( & \boldsymbol{\Phi}_{12} \boldsymbol{\Phi}_{22}^{-1} \boldsymbol{\Phi}_{12}^{T}+\boldsymbol{\Phi}_{13} \boldsymbol{\Phi}_{33}^{-1} \boldsymbol{\Phi}_{13}^{T}+\boldsymbol{\Phi}_{14} \boldsymbol{\Phi}_{44}^{-1} \boldsymbol{\Phi}_{14}^{T} \\
& \left.+\boldsymbol{\Phi}_{15} \boldsymbol{\Phi}_{55}^{-1} \boldsymbol{\Phi}_{15}^{T}\right)
\end{aligned}
$$

Let us consider the following performance function to achieve the attenuation performance of system for all $w(k) \neq$ 0 with zero initial condition:

$$
\begin{aligned}
& J(x(k)) \\
& =E\left\{\sum_{k=0}^{k_{q}}\left(z^{T}(k) \mathbf{S}_{2} z(k)+v^{T}(k) \mathbf{S}_{3} v(k)-2 z^{T}(k) \mathbf{S}_{1} v(k)\right)\right\} \\
& \leq E\left\{\sum _ { k = 0 } ^ { k _ { q } } \left(z^{T}(k) \mathbf{S}_{2} z(k)+v^{T}(k) \mathbf{S}_{3} v(k)\right.\right. \\
& \left.\left.-2 z^{T}(k) \mathbf{S}_{1} v(k)+\Delta V(x(k))\right)\right\} .
\end{aligned}
$$

According to (26), the following inequality can be obtained via (28):

$$
\begin{aligned}
& J(x(k)) \\
& <E \sum_{k=0}^{k_{q}}\left\{\sum_{i=1}^{r} \sum_{j=1}^{r} \widehat{h}_{i} \widehat{h}_{j}\left\{\xi^{T}(k)\left(\Pi_{i j}+\frac{1}{2}\left(\mathbf{W}_{i j}+\mathbf{W}_{j i}\right)\right) \xi(k)\right\}\right\} .
\end{aligned}
$$

Using the Schur complement, one can find the following inequality from (10):

$$
\Pi_{i j}+\frac{1}{2}\left(\mathbf{W}_{i j}+\mathbf{W}_{j i}\right)<0 .
$$

Obviously, if condition (10) is held then (29) is strictly negative that is, $J(x(k))<0$, due to (30). And the following relation can be obtained from $(28)$ according to $J(x(k))<0$ :

$$
E\left\{\sum_{k=0}^{k_{q}}\left(z^{T}(k) \mathbf{S}_{2} z(k)+v^{T}(k) \mathbf{S}_{3} v(k)-2 z^{T}(k) \mathbf{S}_{1} v(k)\right)\right\}
$$$$
<0
$$

or

$$
\begin{gathered}
E\left\{2 \sum_{k=0}^{k_{q}}\left(z^{T}(k) \mathbf{S}_{1} v(k)\right)\right\} \\
>E\left\{\sum_{k=0}^{k_{q}}\left(z^{T}(k) \mathbf{S}_{2} z(k)+v^{T}(k) \mathbf{S}_{3} v(k)\right)\right\} .
\end{gathered}
$$

Since (32) is equivalent to (7) defined in Definition 1, the closed-loop system is passive for all nonzero external disturbance; that is, $v(t) \neq 0$.

Next, we will show that the closed-loop system (5) with all admissible uncertainties is robustly asymptotically stable in the mean square. By assuming $v(t)=0$, the following inequality can be obtained from (30):

$$
\begin{aligned}
\boldsymbol{\Pi}_{i j}<\frac{-1}{2}\{ & \left(\mathbf{C}_{1 i} x(k)+\mathbf{C}_{d i} x(k-\tau(k))\right)^{T} \\
& \times \mathbf{S}_{2}\left(\mathbf{C}_{1 j} x(k)+\mathbf{C}_{d j} x(k-\tau(k))\right) \\
& +\left(\mathbf{C}_{1 j} x(k)+\mathbf{C}_{d j} x(k-\tau(k))\right)^{T} \\
& \left.\times \mathbf{S}_{2}\left(\mathbf{C}_{1 i} x(k)+\mathbf{C}_{d i} x(k-\tau(k))\right)\right\} .
\end{aligned}
$$

Obviously, if $\boldsymbol{S}_{2} \geq 0$ is held, then $\Pi_{i j}<0$. Hence, from (26), the $E\{\Delta V(x(k))\}<0$ can be obtained due to $\Pi_{i j}<0$. In this case, the closed-loop system is robustly asymptotically stable in the mean square from Definition 2. The proof of this theorem is completed.

In Theorem 5, condition (10) simultaneously includes variables $\mathbf{P}_{1}, \mathbf{P}_{1}^{-1}, \mathbf{P}_{5}$, and $\mathbf{P}_{5}^{-1}$ such that (10) is not a strictly 
LMI problem. For applying the LMI technique, let us introduce two new variables, that is, $\mathbf{X}_{1}$ and $\mathbf{X}_{5}$, such that

$$
\mathbf{P}_{1} \mathbf{X}_{1}=\mathbf{I}, \quad \mathbf{P}_{5} \mathbf{X}_{5}=\mathbf{I},
$$

and use $\mathbf{X}_{1}$ and $\mathbf{X}_{5}$ to substitute $\mathbf{P}_{1}^{-1}$ and $\mathbf{P}_{5}^{-1}$ in condition (10), respectively. Based on the cone complementarity technique [34], the following nonlinear minimization problem is proposed instead of the original nonconvex condition (10):

$$
\begin{array}{ll}
\text { Minimize } & \operatorname{tr}\left(\mathbf{P}_{1} \mathbf{X}_{1}+\mathbf{P}_{5} \mathbf{X}_{5}\right) \\
\text { Subject to } & (10)\left(\mathbf{P}_{1}^{-1} \text { and } \mathbf{P}_{5}^{-1}\right. \text { are replaced } \\
& \text { by } \left.\mathbf{X}_{1} \text { and } \mathbf{X}_{5}, \text { resp. }\right), \\
& {\left[\begin{array}{cc}
\mathbf{P}_{1} & \mathbf{I} \\
\mathbf{I} & \mathbf{X}_{1}
\end{array}\right] \geq 0, \quad\left[\begin{array}{cc}
\mathbf{P}_{5} & \mathbf{I} \\
\mathbf{I} & \mathbf{X}_{5}
\end{array}\right] \geq 0 .}
\end{array}
$$

Although the above minimization problem gives suboptimal solutions for original problem (10), it is much easier to solve (35) than the original nonconvex problem. In order to find the feasible solutions of (35), the following algorithm is proposed.

\section{Algorithm 6.}

Step 1. Choose the time delay values $\tau_{\min }, \tau_{\max }$ and performance matrices $\mathbf{S}_{1}, \mathbf{S}_{2} \geq 0$, and $\mathbf{S}_{3}$. Set $t=0$, and find the initial feasible solution set $\left\{\mathbf{P}_{1}, \mathbf{P}_{5}, \mathbf{X}_{1}, \mathbf{X}_{5}\right\}^{t}$ to satisfy condition (10) with $\mathbf{P}_{1}^{-1} \triangleq \mathbf{X}_{1}$ and $\mathbf{P}_{5}^{-1} \triangleq \mathbf{X}_{5}$.

Step 2. Solve the following LMI problem:

$$
\begin{array}{ll}
\text { Minimize } & \operatorname{tr}\left(\mathbf{P}_{1} \mathbf{X}_{1}^{t}+\mathbf{X}_{1} \mathbf{P}_{1}^{t}+\mathbf{P}_{5} \mathbf{X}_{5}^{t}+\mathbf{X}_{5} \mathbf{P}_{5}^{t}\right) \\
\text { Subject to } & (10)\left(\mathbf{P}_{1}^{-1} \text { and } \mathbf{P}_{5}^{-1}\right. \text { are replaced } \\
& \text { by } \left.\mathbf{X}_{1} \text { and } \mathbf{X}_{5}, \text { resp. }\right), \\
& {\left[\begin{array}{cc}
\mathbf{P}_{1} & \mathbf{I} \\
\mathbf{I} & \mathbf{X}_{1}
\end{array}\right] \geq 0, \quad\left[\begin{array}{cc}
\mathbf{P}_{5} & \mathbf{I} \\
\mathbf{I} & \mathbf{X}_{5}
\end{array}\right] \geq 0 .}
\end{array}
$$

Step 3. Substitute the feasible solutions obtained from Step 2 into (10). If condition (10) is satisfied, then go back to Step 2 after increasing the $\tau_{\max }$ until (10) is not satisfied with specified $\tau_{\max }$. In this case, the feasible solutions are obtained and the algorithm can be stopped. Otherwise, go to the next step. Step 4. Set $t=t+1$ and $\left\{\mathbf{P}_{1}, \mathbf{P}_{5}, \mathbf{X}_{1}, \mathbf{X}_{5}\right\}^{t}=\left\{\mathbf{P}_{1}, \mathbf{P}_{5}, \mathbf{X}_{1}, \mathbf{X}_{5}\right\}$; then go to Step 2 .

Remark 7. In order to apply the LMI technique, Algorithm 6 is a useful tool to find the feasible solutions of conditions of Theorem 5. In Algorithm 6, the number of desired unknown variables in fuzzy controller design process is $2 r^{2}+r+8$. From Theorem 3 of [9], one can find that the number of desired unknown variables is $72 r^{3}+7 r^{2}+11 r+6$. Obviously, the number of desired unknown variables of the proposed method is less than that developed in [9].

Remark 8. In [9], the quadratic transformation inequality "W $+\mathbf{W}+\mathbf{P} \geq \mathbf{W P}^{-1} \mathbf{W}$ " is often applied for converting the bilinear matrix inequalities into linear matrix inequalities. During the transformation process, the conservatisms arise to find the solutions of sufficient conditions of Theorems 2 and 3 in [9]. Oppositely, the similar bilinear matrix inequalities are solved via the cone complement technique in this paper. Applying the cone complement technique, the bilinear matrix inequalities are converted into nonstrictly linear matrix inequalities that can be solved by a suboptimal algorithm, that is, Algorithm 6.

Remark 9. In Theorem 5, the free-weighting matrix technique is applied to reduce the conservatism of considered fuzzy controller design problems. By applying the freeweighting matrix technique, more free matrices are added to reduce the conservatism of derived sufficient conditions. However, adding free matrices also increases the computational complexity. In order to balance the incompatible case, it is recommended to use the free-weighting matrix technique as less as possible.

Theorem 5 provides the sufficient conditions (10) to design state-feedback fuzzy controller for guaranteeing robust asymptotical stability and passivity of closed-loop system (5) in mean square. In the following, with the few modifications, Theorem 5 can also be applied to find the outputfeedback gains for structuring the fuzzy controller. Based on (1c), the output-feedback fuzzy controller can also be structured via PDC technique such as

$$
u(k)=\sum_{i=1}^{r} \widehat{h}_{i}\left(\mathbf{K}_{i} y(k)\right) .
$$

Introducing (37) into (1a), the closed-loop system can be obtained as follows:

$$
\begin{aligned}
& x(k+1) \\
& =\sum_{i=1, j=1, p=1}^{r} \widehat{h}_{i} \widehat{h}_{j} \widehat{h}_{p} \\
& \times\left\{\left(\mathbf{g}_{i j p}+\mathbf{H}_{i} \Delta \mathbf{g}_{\text {Rijp }}+\left(\overline{\mathbf{g}}_{i j p}+\overline{\mathbf{H}}_{i} \bar{\Delta} \overline{\mathbf{g}}_{R i j p}\right) \beta(k)\right) \xi(k)\right\} \\
& =\sum_{i=1}^{r} \sum_{j=1}^{r} \sum_{p=1}^{r} \widehat{h}_{i} \widehat{h}_{j} \widehat{h}_{p} \\
& \times\left\{\frac { 1 } { 3 } \left(\mathbf{g}_{i j p}+\mathbf{H}_{i} \Delta \mathbf{g}_{R i j p}+\mathbf{g}_{j p i}\right.\right. \\
& +\mathbf{H}_{j} \Delta \mathbf{g}_{R j p i}+\mathbf{g}_{p i j}+\mathbf{H}_{p} \Delta \mathbf{g}_{R p i j} \\
& +\left(\overline{\mathbf{g}}_{i j p}+\overline{\mathbf{H}}_{i} \bar{\Delta}_{\mathbf{g}_{R i j p}}+\overline{\mathbf{g}}_{j p i}+\overline{\mathbf{H}}_{j} \bar{\Delta} \overline{\mathbf{g}}_{R j p i}\right. \\
& \left.\left.\left.+\overline{\mathbf{g}}_{p i j}+\overline{\mathbf{H}}_{p} \bar{\Delta}_{\mathbf{g}_{R p i j}}\right) \beta(k)\right) \xi(k)\right\},
\end{aligned}
$$

where $\mathbf{g}_{i j p}=\left[\begin{array}{llll}\mathbf{G}_{i j p} & \mathbf{A}_{d i} & \mathbf{E}_{i} & 0_{1 \times 3}\end{array}\right], \overline{\mathbf{g}}_{i j p}=\left[\begin{array}{llll}\overline{\mathbf{G}}_{i j p} & \overline{\mathbf{A}}_{d i} & \overline{\mathbf{E}}_{i} & 0_{1 \times 3}\end{array}\right]$, $\mathbf{g}_{\text {Rijp }}=\left[\begin{array}{llll}\mathbf{R}_{i j p} & \mathbf{R}_{d i} & \mathbf{R}_{E i} & 0_{1 \times 3}\end{array}\right], \overline{\mathbf{g}}_{R i j p}=\left[\begin{array}{llll}\overline{\mathbf{R}}_{i j p} & \overline{\mathbf{R}}_{d i} & \overline{\mathbf{R}}_{E i} & 0_{1 \times 3}\end{array}\right]$, $\mathbf{G}_{i j p}=\mathbf{A}_{i}+\mathbf{B}_{i} \mathbf{K}_{j} \mathbf{C}_{2 p}, \overline{\mathbf{G}}_{i j p}=\overline{\mathbf{A}}_{i}+\overline{\mathbf{B}}_{i} \mathbf{K}_{j} \mathbf{C}_{2 p}, \mathbf{R}_{i j p}=\mathbf{R}_{i}+$ $\mathbf{R}_{B i} \mathbf{K}_{j} \mathbf{C}_{2 p}$, and $\overline{\mathbf{R}}_{i j p}=\overline{\mathbf{R}}_{i}+\overline{\mathbf{R}}_{B i} \mathbf{K}_{j} \mathbf{C}_{2 p}$. 
Theorem 10. Given performance parameters $\mathbf{S}_{1}, \mathbf{S}_{2} \geq 0$, and $\mathbf{S}_{3}$ and values $\tau_{\min }>0$ and $\tau_{\max }>0$, the closed-loop system (38) is robustly asymptotically stable and passive in the sense of mean square, if there exist positive definite matrices $\mathbf{P}_{1}>0, \mathbf{P}_{2}>0, \mathbf{P}_{3}>0, \mathbf{P}_{4}>0$ and $\mathbf{P}_{5}>0$, any matrices $\mathbf{N}_{1}, \mathbf{N}_{2}$ and $\mathbf{N}_{3}$, scalars $\varepsilon_{i j p}>0$ and $\bar{\varepsilon}_{i j p}>0$, and outputfeedback gains $\mathbf{K}_{i}$ such that

$$
\Omega<0 \text { for } i, j, p \leq r,
$$

where

$$
\begin{aligned}
& \boldsymbol{\Omega}=\left[\begin{array}{ccccc}
\boldsymbol{\Omega}_{11} & \boldsymbol{\Omega}_{12} & \boldsymbol{\Omega}_{13} & \boldsymbol{\Omega}_{14} & \boldsymbol{\Omega}_{15} \\
* & \boldsymbol{\Omega}_{22} & 0 & 0 & 0 \\
* & * & \boldsymbol{\Omega}_{33} & 0 & 0 \\
* & * & * & \boldsymbol{\Omega}_{44} & 0 \\
* & * & * & * & \boldsymbol{\Omega}_{55}
\end{array}\right], \\
& \boldsymbol{\Omega}_{11}=\overline{\boldsymbol{\Phi}}+\frac{1}{3}\left(\mathbf{W}_{i i}+\mathbf{W}_{j j}+\mathbf{W}_{i j}\right) \text {, } \\
& \boldsymbol{\Omega}_{12}=\frac{1}{3}\left[\begin{array}{lll}
\boldsymbol{\Omega}_{12 i j p} & \boldsymbol{\Omega}_{12 j p i} & \boldsymbol{\Omega}_{12 p i j}
\end{array}\right], \\
& \boldsymbol{\Omega}_{13}=\sqrt{\tau_{\max }} \mathbf{\Omega}_{12}, \\
& \boldsymbol{\Omega}_{14}=\frac{1}{3}\left[\begin{array}{lll}
\boldsymbol{\Omega}_{14 i j p} & \boldsymbol{\Omega}_{14 j p i} & \boldsymbol{\Omega}_{14 p i j}
\end{array}\right], \quad \boldsymbol{\Omega}_{15}=\sqrt{\tau_{\max }} \boldsymbol{\Omega}_{14}, \\
& \boldsymbol{\Omega}_{22}=\operatorname{diag}\left\{\boldsymbol{\Omega}_{22 i j p}, \boldsymbol{\Omega}_{22 j p i}, \boldsymbol{\Omega}_{22 p i j}\right\}, \\
& \boldsymbol{\Omega}_{33}=\operatorname{diag}\left\{\boldsymbol{\Omega}_{33 i j p}, \boldsymbol{\Omega}_{33 j p i}, \boldsymbol{\Omega}_{33 p i j}\right\} \text {, } \\
& \boldsymbol{\Omega}_{44}=\operatorname{diag}\left\{\boldsymbol{\Omega}_{44 i j p}, \boldsymbol{\Omega}_{44 j p i}, \boldsymbol{\Omega}_{44 p i j}\right\} \text {, } \\
& \boldsymbol{\Omega}_{55}=\operatorname{diag}\left\{\boldsymbol{\Omega}_{55 i j p}, \boldsymbol{\Omega}_{55 j p i}, \boldsymbol{\Omega}_{55 p i j}\right\} \text {, } \\
& \boldsymbol{\Omega}_{12 i j p}=\left[\begin{array}{ll}
\mathbf{f}_{i j p}^{T} & \mathbf{f}_{R i j p}^{T}
\end{array}\right], \quad \boldsymbol{\Omega}_{14 i j p}=\left[\begin{array}{ll}
\overline{\mathbf{f}}_{i j p}^{T} & \overline{\mathbf{f}}_{R i j p}^{T}
\end{array}\right], \\
& \boldsymbol{\Omega}_{33 i j p}=\operatorname{diag}\left\{\varepsilon_{i j p} \mathbf{H}_{i} \mathbf{H}_{i}^{T}-\mathbf{P}_{5}^{-1},-\varepsilon_{i j p} \mathbf{I}\right\}, \\
& \boldsymbol{\Omega}_{22 i j p}=\operatorname{diag}\left\{\varepsilon_{i j p} \mathbf{H}_{i} \mathbf{H}_{i}^{T}-\mathbf{P}_{1}^{-1},-\varepsilon_{i j p} \mathbf{I}\right\} \text {, } \\
& \boldsymbol{\Omega}_{44 i j p}=\operatorname{diag}\left\{\bar{\varepsilon}_{i j p} \overline{\mathbf{H}}_{i} \overline{\mathbf{H}}_{i}^{T}-\mathbf{P}_{1}^{-1},-\bar{\varepsilon}_{i j p} \mathbf{I}\right\} \text {, } \\
& \boldsymbol{\Omega}_{55 i j p}=\operatorname{diag}\left\{\bar{\varepsilon}_{i j p} \overline{\mathbf{H}}_{i} \overline{\mathbf{H}}_{i}^{T}-\mathbf{P}_{5}^{-1},-\bar{\varepsilon}_{i j p} \mathbf{I}\right\} \text {. }
\end{aligned}
$$

With the same Lyapunov function (13), the proof of Theorem 10 can be obtained with similar procedure of proof of Theorem 5 . Hence, the proof of Theorem 10 is omitted here. Although the feasible solutions condition (39) of Theorem 10 cannot be directly obtained by using LMI technique, one can also apply Algorithm 6 by substituting sufficient condition (39) for (10). And then, the feasible solutions can be obtained for satisfying condition (39) and hence the modified algorithm is omitted here.

In the following section, the two numerical examples are provided to apply the proposed fuzzy controller design technique in this paper.

\section{Numerical Examples}

In this section, two numerical examples apply the proposed fuzzy controller design method in this paper. In the first example, the less conservatism of stability criteria in this paper can be shown and demonstrated. On the other hand, in Example 2, both of Theorems 5 and 10 are applied to design the state-feedback fuzzy controller and outputfeedback fuzzy controller, respectively.

Example 1. Referring to [9], the following T-S fuzzy model without multiplicative noise term is proposed to apply the proposed design technique in Theorem 5:

$$
\begin{aligned}
x(k+1)=\sum_{i=1}^{2} \widehat{h}_{i}\left\{\left(\mathbf{A}_{i}\right.\right. & \left.+\Delta \mathbf{A}_{i}\right) x(k) \\
& +\left(\mathbf{A}_{d i}+\Delta \mathbf{A}_{d i}\right) x(k-\tau(k)) \\
& \left.+\left(\mathbf{B}_{i}+\Delta \mathbf{B}_{i}\right) u(k)+\left(\mathbf{E}_{i}+\Delta \mathbf{E}_{i}\right) v(k)\right\}
\end{aligned}
$$

$$
z(k)=\sum_{i=1}^{2} \widehat{h}_{i}\left\{\mathbf{C}_{1 i} x(k)+\mathbf{C}_{d i} x(k-\tau(k))+\mathbf{D}_{i} v(k)\right\}
$$

$$
x(k)=\varphi(k), \quad k=0,-1, \ldots,-\tau(k),
$$

where $\mathbf{A}_{1}=\left[\begin{array}{cc}0.5 & 0.3 \\ 0.1 & 1\end{array}\right], \mathbf{A}_{2}=\left[\begin{array}{cc}-0.5 & 0.3 \\ 0.1 & 1\end{array}\right], \mathbf{A}_{d 1}=\left[\begin{array}{cc}-0.05 & 0.1 \\ 0 & 0.05\end{array}\right]$, $\mathbf{B}_{1}=\left[\begin{array}{cc}1 & 0 \\ 0 & 0.5\end{array}\right], \mathbf{E}_{1}=\left[\begin{array}{c}-0.5 \\ 0\end{array}\right], \mathbf{A}_{d 2}=-\mathbf{A}_{d 1}, \mathbf{B}_{2}=\mathbf{B}_{1}, \mathbf{E}_{2}=$ $-\mathbf{E}_{1}, \mathbf{C}_{11}=\left[\begin{array}{ll}-0.05 & 0\end{array}\right], \mathbf{C}_{12}=-\mathbf{C}_{11}, \mathbf{H}_{i}=\left[\begin{array}{cc}-0.05 & 0.1 \\ 0.1 & 0\end{array}\right], \mathbf{R}_{i}=$ $\left[\begin{array}{cc}0 & 0.2 \\ 0 & 0\end{array}\right], \mathbf{R}_{d i}=\left[\begin{array}{ll}0 & 0 \\ 0 & 0.1\end{array}\right], \mathbf{R}_{B i}=\mathbf{R}_{E i}=0, \mathbf{C}_{d i}=\left[\begin{array}{ll}0 & 0.2\end{array}\right]$, and $\mathbf{D}_{i}=0.1$ for $i=1,2$.

And the membership function of (43a), (43b), and (43c) is chosen as $\widehat{h}_{1}=\left(1-2 x_{1}(k)\right) / 2$ and $\widehat{h}_{2}=1-\widehat{h}_{1}$. For comparing the proposed method with that developed in [9], the passivity performance is chosen as $H_{\infty}$ performance constraint by setting $\mathbf{S}_{1} \triangleq 0, \mathbf{S}_{2} \triangleq \mathbf{I}$, and $\mathbf{S}_{3} \triangleq-\gamma^{2} \mathbf{I}$. For finding maximum allowable $\tau_{\text {max }}$, let us study different cases with $\tau_{\min }=2, \tau_{\min }=5$, and $\tau_{\min }=10$. From Table 1 , one can find that the allowed upper bound of delay $\tau_{\max }$ controlled by the proposed design method is bigger than that of [9]. It means that the proposed design method can provide bigger maximum delay bound than the approach developed in [9]. Besides, the smaller $H_{\infty}$ performance level $\gamma$ can be found by using the proposed design method. It should be noted that the stability criterion of this paper possesses less conservatism than that proposed in [9].

Next, we apply the proposed design techniques to find both of state-feedback fuzzy controller and output-feedback fuzzy controller for nonlinear delay Hénon system.

Example 2. In this example, the nonlinear delay Hénon system is proposed to apply the proposed fuzzy controller design techniques. Referring to [10], the nonlinear delay Hénon system with external disturbance can be proposed as follows:

$$
\begin{aligned}
x_{1}(k+1)= & \left(0.8 x_{1}(k)+0.2 x_{1}(k-\tau(k))\right)^{2} \\
& +0.3 x_{2}(k)+1.4+\tilde{u}(k),
\end{aligned}
$$


TABLE 1: Comparisons between proposed method and [9].

\begin{tabular}{lcccccc}
\hline$\tau_{\min }$ & Design method & $\tau_{\max }=12$ & $\tau_{\max }=13$ & $\tau_{\max }=14$ & $\tau_{\max }=15$ & 0.5909 \\
\multirow{2}{*}{10} & $\gamma$ in [9] & 0.3408 & 0.4085 & 0.4897 & 0.3515 & Infeasible \\
& $\gamma$ in Theorem 5 & 0.3351 & 0.338 & 0.3481 & 0.3595 \\
\hline \multirow{2}{*}{5} & $\gamma$ in [9] & 0.4882 & 0.806 & 0.6963 & 0.8468 & Infeasible \\
& $\gamma$ in Theorem 5 & 0.3691 & 0.3712 & 0.383 & 0.394 & 0.4012 \\
\hline \multirow{2}{*}{2} & $\gamma$ in [9] & 0.5818 & 0.6932 & 0.8369 & Infeasible & Infeasible \\
& $\gamma$ in Theorem 5 & 0.4124 & 0.4087 & 0.5124 & 0.598 & 0.612 \\
\hline
\end{tabular}

$$
\begin{gathered}
x_{2}(k+1)=0.8 x_{1}(k)+0.2 x_{1}(k-\tau(k))+0.1 v(k), \\
z(k)=0.8 x_{1}(k)+0.2 x_{1}(k-\tau(k))+v, \\
y(k)=x_{1}, \\
x(k)=\varphi(k), \quad k=0,-1, \ldots,-\tau(k),
\end{gathered}
$$

where $v(k)$ is chosen as a zero-mean white noise with variance 0.5 . Based on the fuzzy modeling in [10], the considered discrete uncertain stochastic T-S fuzzy model of (44a), (44b), (44c), (44d), and (44e) can be obtained by adding the uncertainties and stochastic behaviors

$$
\begin{gathered}
x(k+1) \\
=\sum_{i=1}^{2} \widehat{h}_{i}\left\{\left(\mathbf{A}_{i}+\Delta \mathbf{A}_{i}\right) x(k)\right. \\
+\left(\mathbf{A}_{d i}+\Delta \mathbf{A}_{d i}\right) x(k-\tau(k)) \\
+\left(\mathbf{B}_{i}+\Delta \mathbf{B}_{i}\right) u(k)+\mathbf{E}_{i} v(k) \\
\left.+\left(\overline{\mathbf{A}}_{i} x(k)+\overline{\mathbf{A}}_{d i} x(k-\tau(k))+\overline{\mathbf{B}}_{i} u(k)\right) \beta(k)\right\} \\
\\
z(k)=\sum_{i=1}^{2} \widehat{h}_{i}\left\{\mathbf{C}_{1 i} x(k)+\mathbf{D}_{i} v(k)\right\}, \\
y(k)=\sum_{i=1}^{2} \widehat{h}_{i}\left\{\mathbf{C}_{2 i} x(k)\right\},
\end{gathered}
$$

where $u(k)=1.4+\widetilde{u}(k)$, premise variable $\theta(k)=0.8 x_{1}(k)+$ $0.2 x_{1}(k-\tau(k))$, membership function $\widehat{h}_{1}=(1 / 2)(1-(\theta(k) /$ 2)) and $\widehat{h}_{2}=1-\widehat{h}_{1}, \mathbf{A}_{1}=\left[\begin{array}{cc}1.6 & 0.3 \\ 0.8 & 0\end{array}\right], \mathbf{A}_{2}=\left[\begin{array}{cc}-1.8 & 0.3 \\ 0.8 & 0\end{array}\right], \mathbf{A}_{d 1}=$ $\left[\begin{array}{ll}0.4 & 0 \\ 0.2 & 0\end{array}\right], \mathbf{A}_{d 1}=\left[\begin{array}{rr}-0.4 & 0 \\ 0.2 & 0\end{array}\right], \mathbf{B}_{i}=\left[\begin{array}{l}1 \\ 0\end{array}\right], \mathbf{E}_{i}=\left[\begin{array}{c}0 \\ 0.1\end{array}\right], \mathbf{C}_{1 i}=\left[\begin{array}{ll}0.8 & 0\end{array}\right]$, $\mathbf{C}_{d i}=\left[\begin{array}{ll}0.2 & 0\end{array}\right], \mathbf{C}_{2 i}=\left[\begin{array}{ll}1 & 0\end{array}\right], \mathbf{D}_{i}=1, \overline{\mathbf{A}}_{i}=0.1 \mathbf{A}_{i}, \overline{\mathbf{A}}_{d i}=$

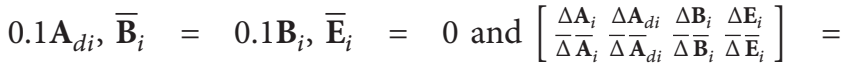

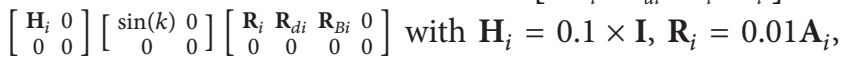
and $\mathbf{R}_{d i}=0.01 \mathbf{A}_{d i}$ and $\mathbf{R}_{B i}=0.01 \mathbf{B}_{i}$, for $i=1,2$.

In the following, both design methods in Theorems 5 and 10 will be applied to design the PDC-based fuzzy controller in the terms of (4) and (37), respectively, such that closed-loop system is robustly asymptotically stable and passive in mean square.
Case A: State-Feedback. Through Theorem 5 and Algorithm 6 of this paper, the following feasible solutions can be found with the range of time-varying delay between $\tau_{\max }=$ 5 and $\tau_{\min }=1$ and chosen passivity (7) with $\mathbf{S}_{1} \triangleq \mathbf{I}, \mathbf{S}_{2} \triangleq$ 0.8 , and $\mathbf{S}_{3} \triangleq 0.8$ :

$$
\begin{aligned}
& \mathbf{P}_{1}=\left[\begin{array}{cc}
30.3351 & -0.3962 \\
-0.3962 & 0.9253
\end{array}\right], \quad \mathbf{P}_{2}=\left[\begin{array}{cc}
0.008 & 0.0001 \\
0.0001 & 0.0029
\end{array}\right] \\
& \mathbf{P}_{3}=\left[\begin{array}{cc}
0.008 & 0.0001 \\
0.0001 & 0.0029
\end{array}\right], \quad \mathbf{P}_{4}=\left[\begin{array}{cc}
5.6071 & -0.0167 \\
-0.0167 & 0.0003
\end{array}\right] \text {, } \\
& \mathbf{P}_{5}=\left[\begin{array}{ll}
0.2488 & 0.0858 \\
0.0858 & 0.1773
\end{array}\right] \text {, } \\
& \mathbf{N}_{1}=\left[\begin{array}{cc}
-3.2735 & -0.011 \\
-0.0111 & -3.3647
\end{array}\right] \times 10^{3} \text {, } \\
& \mathbf{N}_{2}=\left[\begin{array}{ll}
3.2735 & 0.0111 \\
0.0111 & 3.3647
\end{array}\right] \times 10^{3}, \\
& \mathbf{N}_{3}=\left[\begin{array}{ll}
3.2735 & 0.0111 \\
0.0111 & 3.3647
\end{array}\right] \times 10^{3} \text {, } \\
& \varepsilon_{11}=0.0127, \quad \varepsilon_{22}=0.0246, \\
& \mathbf{F}_{1}=\left[\begin{array}{ll}
-1.5878 & -0.2669
\end{array}\right], \quad \mathbf{F}_{2}=\left[\begin{array}{ll}
1.6623 & -0.306
\end{array}\right] .
\end{aligned}
$$

From PDC concept, the fuzzy controller can be established with sublinear state-feedback gains in (46) and the membership function, such as

$$
u(k)=\sum_{i=1}^{3} \widehat{h}_{i}\left(\mathbf{F}_{i} x(k)\right)
$$

With (47), the responses of system (44a), (44b), (44c), (44d), and (44e) by adding the uncertainties and stochastic behaviors are stated in Figure 1 with initial condition $x(0)=$ $\left[\begin{array}{ll}1 & 0\end{array}\right]^{T}$. And the time delay effect is chosen by random block in Simulink of MATLAB and bounded as $1 \leq \tau(k) \leq 5$ in this case. Based on the simulation results in this case, the attenuation performance can be checked by the following equation:

$$
\frac{0.8 E\left\{\sum_{k=0}^{k_{q}} z^{T}(k) z(k)+\sum_{k=0}^{k_{q}} v^{T}(k) v(k)\right\}}{E\left\{2 \sum_{k=0}^{k_{q}} z^{T}(k) v(k)\right\}}=0.8637 .
$$




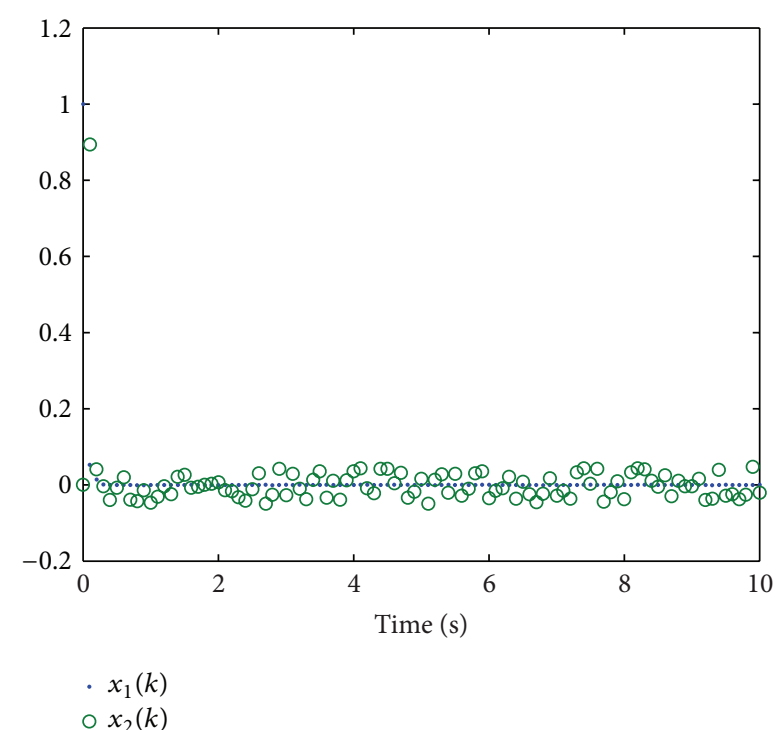

FIGURE 1: State responses driven by fuzzy controller (47): Case A of Example 2.

Obviously, because the radio value of (48) is smaller than one, the chosen passivity inequality can be satisfied with the simulation results of this case. Hence, from (48) and Figure 1, the robust asymptotical stability and passivity of system (45a), (45b), and (45c) by adding the uncertainties and stochastic behaviors can be achieved by design fuzzy controller (47). Next, the case of output-feedback controller design for the same considered system will be shown.

Case B: Output-Feedback. Considering the system (45a), (45b), and (45c) with $\tau_{\max }=2$ and $\tau_{\min }=1$, the following feasible solutions are found to satisfying the Theorem 10 by using modified Algorithm 6:

$$
\begin{gathered}
\mathbf{P}_{1}=\left[\begin{array}{cc}
6.8827 & -1.2065 \\
-1.2065 & 2.0775
\end{array}\right], \\
\mathbf{P}_{2}=\left[\begin{array}{cc}
0.2406 & -0.0869 \\
-0.0869 & 0.1647
\end{array}\right] \times 10^{-3}, \\
\mathbf{P}_{3}=\left[\begin{array}{cc}
0.2406 & -0.0869 \\
-0.0869 & 0.1647
\end{array}\right] \times 10^{-3}, \\
\mathbf{P}_{4}=\left[\begin{array}{cc}
1.9831 & -0.3483 \\
-0.3483 & 0.0614
\end{array}\right], \quad \mathbf{P}_{5}=\left[\begin{array}{ll}
1.4313 & 0.7436 \\
0.7436 & 0.6632
\end{array}\right], \\
\mathbf{N}_{1}=\left[\begin{array}{cc}
-3.2772 & 1.1479 \\
1.016 & -4.2002
\end{array}\right] \times 10^{3}, \\
\mathbf{N}_{2}=\left[\begin{array}{cc}
3.2776 & -1.1483 \\
-1.0161 & 4.2003
\end{array}\right] \times 10^{3}, \\
\mathbf{N}_{3}=\left[\begin{array}{cc}
3.2777 & -1.1483 \\
-1.0156 & 4.1998
\end{array}\right] \times 10^{3},
\end{gathered}
$$

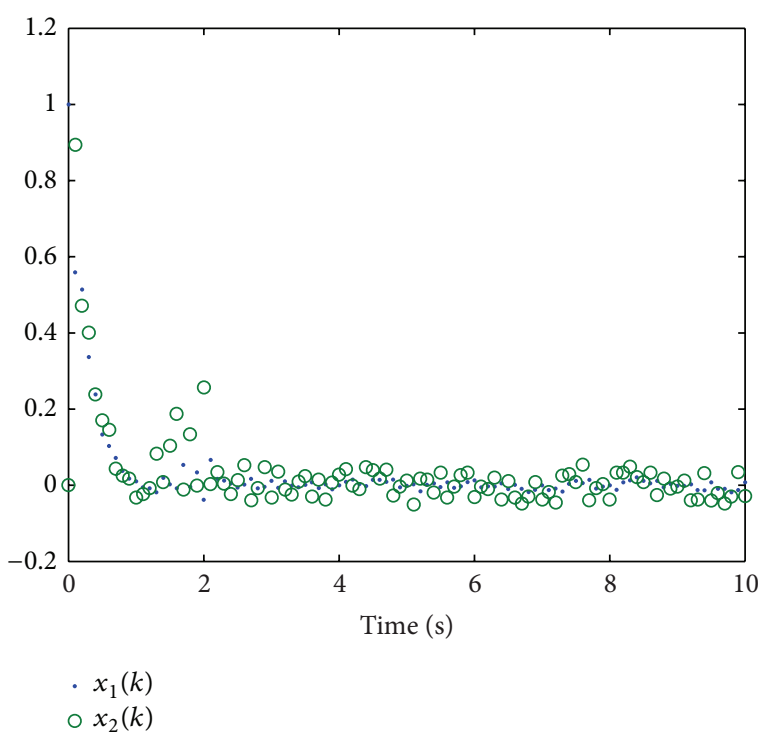

Figure 2: State responses driven by fuzzy controller (50): Case B of Example 2.

$$
\begin{aligned}
\varepsilon_{11}=0.0302, & \varepsilon_{22}=0.0573, \\
\mathbf{K}_{1}=-2.0142, & \mathbf{K}_{2}=2.4929 .
\end{aligned}
$$

With the sublinear output-feedback gains, the PDC-based fuzzy controller can be obtained as follows:

$$
u(k)=\sum_{i=1}^{r} \widehat{h}_{i}\left(\mathbf{K}_{i} y(k)\right) .
$$

In Figure 2, the responses of considered system with fuzzy controller (50) are shown with initial condition $x(0)=$ $\left[\begin{array}{ll}1 & 0\end{array}\right]^{T}$ and random time-varying delay which bounded as $1 \leq \tau(k) \leq 2$. And the attenuation performance of the system can be checked by the following equation:

$$
\frac{0.8 E\left\{\sum_{k=0}^{k_{q}} z^{T}(k) z(k)+\sum_{k=0}^{k_{q}} v^{T}(k) v(k)\right\}}{E\left\{2 \sum_{k=0}^{k_{q}} z^{T}(k) v(k)\right\}}=0.9383
$$

Since the value of (51) is smaller than one, the passivity of system can be achieved by the fuzzy controller (50). From (51) and Figure 2, (45a), (45b), and (45c) with design fuzzy controller are robustly asymptotically stable and passive in the mean square.

\section{Conclusion}

The fuzzy controller design problems of discrete uncertain $\mathrm{T}$ S fuzzy systems with interval time-varying delay and multiplicative noise were discussed and investigated in this paper. With the free-weighting matrices technique and discrete type Jensen inequality, the less conservative stability criterion was derived by applying Lyapunov function. Although the 
derived conditions were not strictly convex problems, the cone complementarity method provided a suboptimal technique to solve it with the LMI technique. Through the proposed design method, the PDC-based fuzzy controller can be established by both of state-feedback and output-feedback schemes for guaranteeing the robust asymptotical stability and passivity constraints. Finally, two numerical examples have been provided to demonstrate the effectiveness and usefulness of the proposed design methods.

\section{Acknowledgments}

The authors would like to express their sincere gratitude to the anonymous reviewers who gave them some constructive comments, criticisms, and suggestions. This work was supported by the National Science Council of the Republic of China under Contract NSC102-2221-E-019-051.

\section{References}

[1] C. Peng and M.-R. Fei, "An improved result on the stability of uncertain T-S fuzzy systems with interval time-varying delay," Fuzzy Sets and Systems, vol. 212, no. 2, pp. 97-109, 2013.

[2] G. Wei, G. Feng, and Z. Wang, "Robust $H_{\infty}$ control for discrete-time fuzzy systems with infinite-distributed delays," IEEE Transactions on Fuzzy Systems, vol. 17, no. 1, pp. 224-232, 2009.

[3] Z.-G. Wu, P. Shi, H. Su, and J. Chu, "Reliable $H_{\infty}$ control for discrete-time fuzzy systems with infinite-distributed delay," IEEE Transactions on Fuzzy Systems, vol. 20, no. 1, pp. 22-31, 2012.

[4] P. L. Liu, "New results on stability analysis for time-varying delay systems with non-linear perturbations," ISA Transactions, vol. 52, no. 2, pp. 215-222, 2013.

[5] S. Boyd, L. El Ghaoui, E. Feron, and V. Balakrishnan, Linear Matrix Inequalities in System and Control Theory, vol. 15 of SIAM Studies in Applied Mathematics, SIAM, Philadelphia, Pa, USA, 1994.

[6] M. Chen, G. Feng, H. Ma, and G. Chen, "Delay-dependent $H_{\infty}$ filter design for discrete-time fuzzy systems with time-varying delays," IEEE Transactions on Fuzzy Systems, vol. 17, no. 3, pp. 604-616, 2009.

[7] H. Zhang and C. Dang, "Piecewise $H_{\infty}$ controller design of uncertain discrete-time fuzzy systems with time delays," IEEE Transactions on Fuzzy Systems, vol. 16, no. 6, pp. 1649-1655, 2008.

[8] L. Wu, X. Su, P. Shi, and J. Qiu, "A new approach to stability analysis and stabilization of discrete-time T-S fuzzy timevarying delay systems," IEEE Transactions on Systems, Man, and Cybernetics, Part B, vol. 41, no. 1, pp. 273-286, 2011.

[9] B. Zhang and $\mathrm{S}$. Xu, "Delay-Dependent Robust $H_{\infty}$ control for uncertain discrete-time fuzzy systems with time-varying delays," IEEE Transactions on Fuzzy Systems, vol. 17, no. 4, pp. 809-823, 2009.

[10] H. Gao, X. Liu, and J. Lam, "Stability analysis and stabilization for discrete-time fuzzy systems with time-varying delay," IEEE Transactions on Systems, Man, and Cybernetics, Part B, vol. 39, no. 2, pp. 306-317, 2009.

[11] S.-M. Wu, C.-C. Sun, H.-Y. Chung, and W.-J. Chang, "Discrete $\mathrm{H}_{2} / \mathrm{H}_{\infty}$ nonlinear controller design based on fuzzy region concept and Takagi-Sugeno fuzzy framework," IEEE Transactions on Circuits and Systems. I. Regular Papers, vol. 53, no. 12, pp. 2838-2848, 2006.

[12] P. Vadivel, R. Sakthivel, K. Mathiyalagan, and P. Thangaraj, "Robust stabilisation of non-linear uncertain Takagi-Sugeno fuzzy systems by $H_{\infty}$ control," IET Control Theory \& Applications, vol. 6, no. 16, pp. 2556-2566, 2012.

[13] A. H. Besheer, H. M. Emara, and M. M. Abdel Aziz, "Fuzzybased output-feedback $H_{\infty}$ control for uncertain nonlinear systems: an LMI approach," IET Control Theory \& Applications, vol. 1, no. 4, pp. 1176-1185, 2007.

[14] J. Dong and G.-H. Yang, " $H_{\infty}$ control for fast sampling discretetime singularly perturbed systems," Automatica, vol. 44, no. 5, pp. 1385-1393, 2008.

[15] B. Zhang, W. X. Zheng, and S. Xu, "Passivity analysis and passive control of fuzzy systems with time-varying delays," Fuzzy Sets and Systems, vol. 174, no. 1, pp. 83-98, 2011.

[16] A. Bemporad, G. Bianchini, and F. Brogi, "Passivity analysis and passification of discrete-time hybrid systems," IEEE Transactions on Automatic Control, vol. 53, no. 4, pp. 1004-1009, 2008.

[17] W.-J. Chang, C.-C. Ku, and P.-H. Huang, "Robust fuzzy control for uncertain stochastic time-delay Takagi-Sugeno fuzzy models for achieving passivity," Fuzzy Sets and Systems, vol. 161, no. 15, pp. 2012-2032, 2010.

[18] R. Lozano, B. Brogliato, O. Egeland, and B. Maschke, Dissipative Systems Analysis and Control Theory and Applications, Communications and Control Engineering Series, Springer, London, UK, 2000.

[19] G. Eli, S. Uri, and Y. Isaac, $H_{\infty}$ Control and Estimation of StateMultiplicative Linear Systems, vol. 318 of Lecture Notes in Control and Information Sciences, Springer, London, UK, 2005.

[20] H. Gao, J. Lam, and Z. Wang, "Discrete bilinear stochastic systems with time-varying delay: stability analysis and control synthesis," Chaos, Solitons \& Fractals, vol. 34, no. 2, pp. 394-404, 2007.

[21] S. Xu, J. Lam, and T. Chen, "Robust $H_{\infty}$ control for uncertain discrete stochastic time-delay systems," Systems \& Control Letters, vol. 51, no. 3-4, pp. 203-215, 2004.

[22] Q. Gao, G. Feng, Y. Wang, and J. Qiu, "Universal fuzzy models and universal fuzzy controllers for stochastic nonaffine nonlinear systems," IEEE Transactions on Fuzzy Systems, vol. 21, no. 2, pp. 328-341, 2013.

[23] B. Zhang, S. Xu, G. Zong, and Y. Zou, "Delay-dependent stabilization for stochastic fuzzy systems with time delays," Fuzzy Sets and Systems, vol. 158, no. 20, pp. 2238-2250, 2007.

[24] H. Huang and D. W. C. Ho, "Delay-dependent robust control of uncertain stochastic fuzzy systems with time-varying delay," IET Control Theory \& Applications, vol. 1, no. 4, pp. 1075-1085, 2007.

[25] X. Liu and H. Zhang, "Delay-dependent robust stability of uncertain fuzzy large-scale systems with time-varying delays," Automatica, vol. 44, no. 1, pp. 193-198, 2008.

[26] C.-C. Ku, P.-H. Huang, and W.-J. Chang, "Observer-based robust fuzzy controller design for uncertain stochastic T-S fuzzy model with passivity performance," in Proceedings of the 48th IEEE Conference on Decision and Control Held Jointly with the 28th Chinese Control Conference (CDC/CCC '09), pp. 77837788, Shanghai, China, December 2009.

[27] C.-S. Tseng, "Robust fuzzy filter design for a class of nonlinear stochastic systems," IEEE Transactions on Fuzzy Systems, vol. 15, no. 2, pp. 261-274, 2007. 
[28] X. Su, P. Shi, L. Wu, and S. K. Nguang, "Induced $l_{2}$ filtering of fuzzy stochastic systems with time-varying delays," IEEE Transactions on Cybernetics, vol. 43, no. 4, pp. 1251-1264, 2013.

[29] Y. Tang, J.-A. Fang, M. Xia, and X. Gu, "Synchronization of Takagi-Sugeno fuzzy stochastic discrete-time complex networks with mixed time-varying delays," Applied Mathematical Modelling. Simulation and Computation for Engineering and Environmental Systems, vol. 34, no. 4, pp. 843-855, 2010.

[30] W.-J. Chang, S.-S. Jheng, and C.-C. Ku, "Fuzzy control with robust and passive properties for discrete-time Takagi-Sugeno fuzzy systems with multiplicative noises," Proceedings of the Institution of Mechanical Engineers. Part I: Journal of Systems and Control Engineering, vol. 226, no. 4, pp. 476-485, 2012.

[31] W. J. Chang, M. W. Chen, and C. C. Ku, "Passive fuzzy controller design for discrete ship steering systems via Takagi-Sugeno fuzzy model with multiplicative noises," Journal of Marine Science and Technology, vol. 21, no. 2, pp. 159-165, 2013.

[32] Y. Niu and D. W. C. Ho, "Stabilization of discrete-time stochastic systems via sliding mode technique," Journal of the Franklin Institute, vol. 349, no. 4, pp. 1497-1508, 2012.

[33] X.-L. Zhu and G.-H. Yang, "Jensen inequality approach to stability analysis of discrete-time systems with time-varying delay," in Proceedings of the American Control Conference (ACC '08), pp. 1644-1649, June 2008.

[34] L. El Ghaoui, F. Oustry, and M. AitRami, "A cone complementarity linearization algorithm for static output-feedback and related problems," IEEE Transactions on Automatic Control, vol. 42, no. 8, pp. 1171-1176, 1997. 


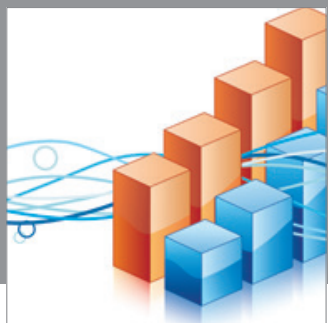

Advances in

Operations Research

mansans

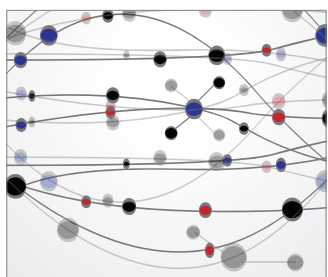

The Scientific World Journal
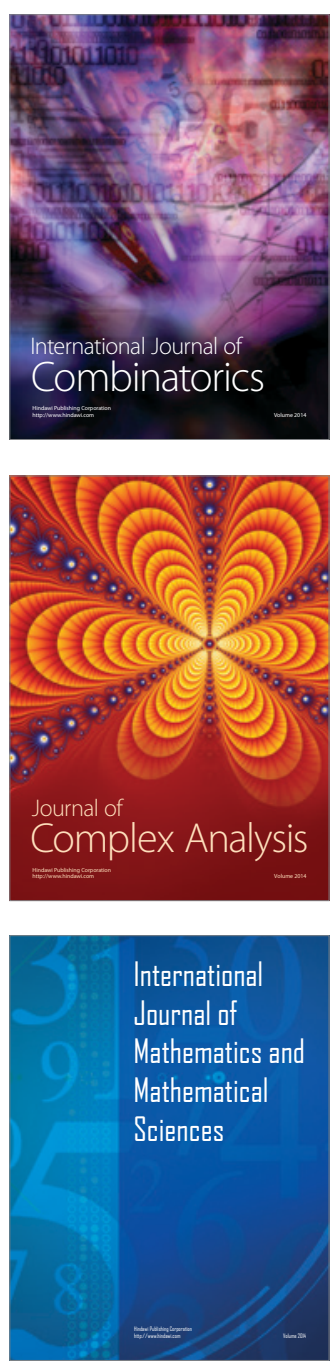
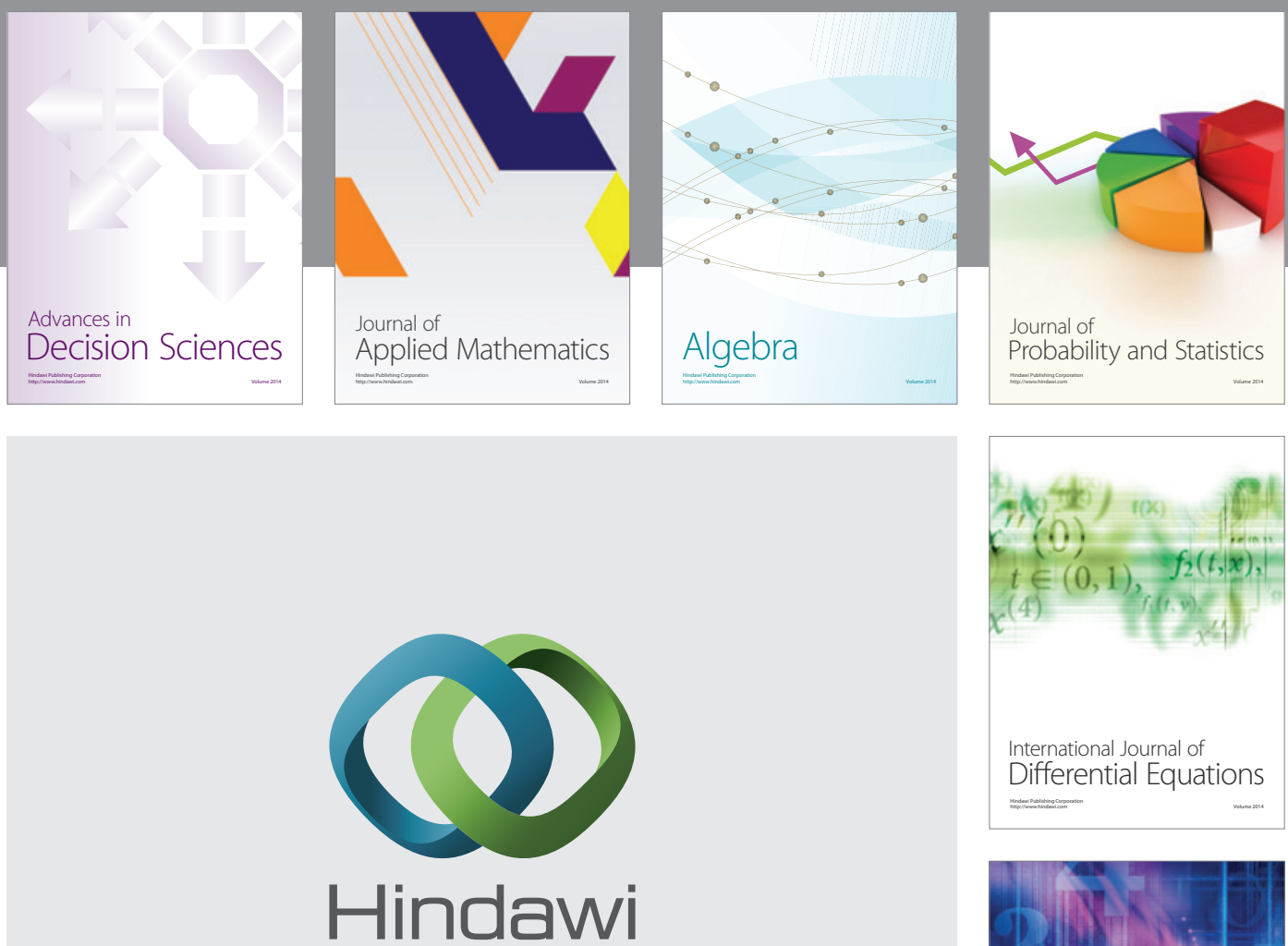

Submit your manuscripts at http://www.hindawi.com
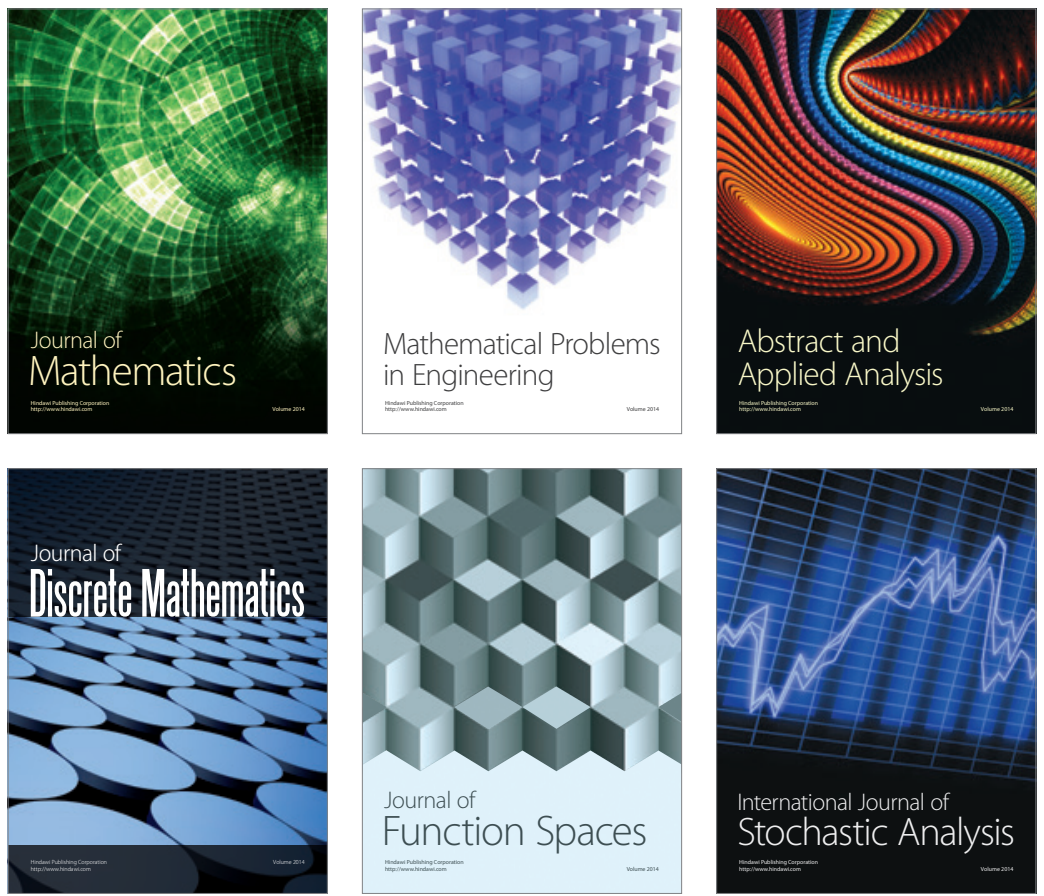

Journal of

Function Spaces

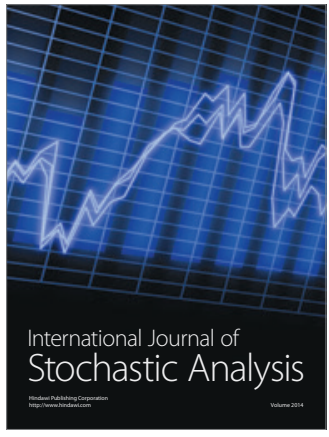

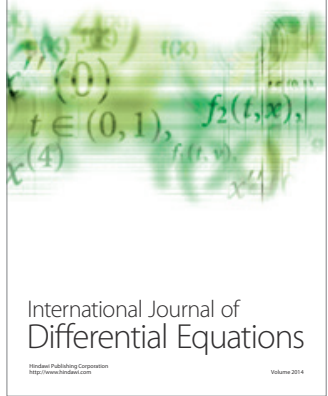
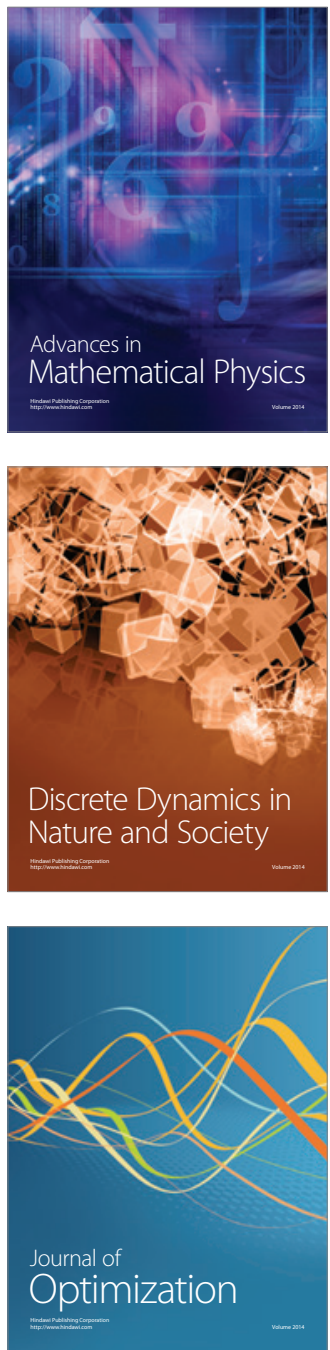